\title{
Conformal Toda theory with a boundary
}

\author{
Vladimir Fateev ${ }^{a, b}$ and Sylvain Ribault ${ }^{a}$ \\ ${ }^{a}$ Laboratoire de Physique Théorique et Astroparticules, UMR5207 CNRS-UM2, \\ Université Montpellier II, Place E. Bataillon, 34095 Montpellier Cedex 05, France \\ ${ }^{b}$ Landau Institute for Theoretical Physics, Academy of Sciences of Russia, \\ Kosygina Str. 2, 117334 Moscow, Russia \\ E-mail: vladimir.fateev@lpta.univ-montp2.fr, sribault@um2.fr
}

ABSTRACT: We investigate $s \ell_{n}$ conformal Toda theory with maximally symmetric boundaries. There are two types of maximally symmetric boundary conditions, due to the existence of an order two automorphism of the $W_{n \geq 3}$ algebra. In one of the two cases, we find that there exist D-branes of all possible dimensions $0 \leq d \leq n-1$, which correspond to partly degenerate representations of the $W_{n}$ algebra. We perform classical and conformal bootstrap analyses of such D-branes, and relate these two approaches by using the semiclassical light asymptotic limit. In particular we determine the bulk one-point functions. We observe remarkably severe divergences in the annulus partition functions, and attribute their origin to the existence of infinite multiplicities in the fusion of representations of the $W_{n \geq 3}$ algebra. We also comment on the issue of the existence of a boundary action, using the calculus of constrained functional forms, and derive the generating function of the Bäcklund transformation for $s \ell_{3}$ Toda classical mechanics, using the minisuperspace limit of the bulk one-point function.

Keywords: Integrable Equations in Physics, D-branes, Conformal and W Symmetry

ARXIV EPRINT: 1007.1293 


\section{Contents}

1 Introduction 2

$2 W_{n}$ algebras and their representations 3

2.1 Representation theory of $s \ell_{n}$ Lie algebras 3

2.2 Representation theory of $W_{n}$ algebras 5

$\begin{array}{lll}2.3 & \text { Fusion multiplicity } & 7\end{array}$

2.4 Lagrangian formulation 9

3 Solutions of the Toda equations on a disc $\quad 10$

$\begin{array}{ll}3.1 \text { Case of Liouville theory } & 10\end{array}$

$\begin{array}{ll}3.2 \text { Case of } s \ell_{3} \text { Toda theory } & 12\end{array}$

$\begin{array}{lll}3.3 & \text { Boundary condition } W-\bar{W}=0 & 12\end{array}$

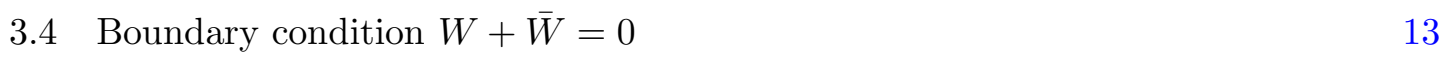

4 Conformal bootstrap study of $s \ell_{3}$ Toda theory with $W+\bar{W}=0 \quad 15$

$\begin{array}{ll}4.1 \text { Continuous D-branes } & 16\end{array}$

$\begin{array}{lll}4.2 & \text { Degenerate D-branes } & 19\end{array}$

$\begin{array}{lll}4.3 & \text { Modular bootstrap analysis } & 22\end{array}$

4.4 Continuous boundary spectra 23

5 Light asymptotic limits of some correlation functions $\quad \mathbf{2 4}$

$\begin{array}{ll}5.1 \text { Case of Liouville theory } & 25\end{array}$

5.2 Case of $s \ell_{3}$ Toda theory with $W-\bar{W}=0 \quad 27$

5.3 Case of $s \ell_{3}$ Toda theory with $W+\bar{W}=0 \quad 30$

6 Conclusion $\quad 33$

A Minisuperspace limits of some correlation functions $\quad 34$

$\begin{array}{lll}\text { A.1 Case of Liouville theory } & 35\end{array}$

A.2 Case of $s \ell_{3}$ Toda theory with $W+\bar{W}=0 \quad 36$

B On the existence of a boundary action in $s \ell_{3}$ Toda theory $\quad 37$

B.1 Boundary conditions as functional one-forms 38

$\begin{array}{lll}\text { B.2 Technical interlude: functional calculus } & 38\end{array}$

B.3 Existence of the boundary action if $W-\bar{W}=0 \quad 40$

B.4 No boundary action if $W+\bar{W}=0 \quad 41$ 


\section{Introduction}

There are good reasons for studying $s \ell_{n}$ conformal Toda theories, as in principle these non-rational two-dimensional conformal field theories have all the usual applications of twodimensional CFTs, applications to quantum gravity, string theory and critical phenomena. (See [1] for more details and references.) In particular, the simplest and well-studied case of Liouville theory $(n=2)$ is an essential tool in the study of non-critical string theories and two-dimensional quantum gravity. And the study of Liouville theory with a boundary plays an important role in the understanding of non-critical open strings and of the corresponding D-branes, which account for the non-perturbative effects in non-critical string theory. The other cases $(n \geq 3)$ are directly related to the so-called $W$-strings and $W$-gravity theories, whose names come from the $W_{n}$ symmetry algebra of $s \ell_{n}$ conformal Toda theory. And the non-perturbative effects in $W$ string theory are expected to be due to D-branes, which can be technically described using conformal Toda theory with a boundary. In addition to such applications, another motivation for investigating the higher Toda theories is their beautiful, intricate and challenging nature, which suggests that their study can reveal qualitatively new structures and phenomena in two-dimensional conformal field theory.

The $W_{n}$ algebra, which is an extension of the Virasoro algebra, was discovered $[2,3]$ soon after the seminal work of Belavin, Polyakov and Zamolodchikov on two-dimensional CFTs [4], and rational CFTs with $W_{n}$ symmetries were then constructed [3, 5]. The study of $s \ell_{n}$ conformal Toda theories, which are non-rational CFTs with $W_{n}$ symmetries, is much more recent $[1,6]$. The case of Liouville theory had to be studied first, and $s \ell_{n \geq 3}$ Toda theory is considerably more complicated than Liouville theory. The reasons for these extra complications can be found in the properties of the $W_{n}$ algebras, as we will demonstrate.

Our most powerful tool in the study of non-rational CFTs is the conformal bootstrap method, which purposes to determine all correlation functions once the spectrum of the theory is given, and the $W_{n}$ symmetry of the theory is assumed. So far this has been achieved only in the case of Liouville theory; however this is in principle doable also in $s \ell_{n \geq 3}$ Toda theories. The conformal bootstrap equations for say the three-point functions are vastly overdetermined, the problem is to find closed subsystems of manageable numbers of equations. In the present article we will achieve this in the case of the one-point function in the presence of a boundary. Introducing a boundary in the two-dimensional space on which our field theory lives of course makes the theory more complicated, but the advantage is that simple correlation functions like the one point function, which has to vanish in the absence of a boundary, now become interesting observables.

In the case of CFTs with boundaries, the fundamental relations between the properties of the symmetry algebra and the physical observables of the theory were discovered by Cardy [7], and we will refer to them as "Cardy's ideas". First of all, maximally symmetric D-branes are related to the representations of the symmetry algebra which appear in the bulk spectrum. Then, the spectrum of open strings with their ends on two D-branes is given by the fusion product of the two corresponding representations. We will find that these properties mostly hold in $s \ell_{n}$ conformal Toda theory, in the cases where we can determine the relevant objects. There will be restrictions, some of which were already observed in 
the case of Liouville theory: there exist not only continuous D-branes associated to the continuous representations which do appear in the bulk spectrum, but also discrete Dbranes associated to degenerate representations which do not.

So we will begin with a study of $W_{n}$ algebras and their representations (section 2), where we will emphasize the features which will play an important role in $s \ell_{n}$ Toda theory: the properties of the characters, the existence of an order 2 automorphism, the existence of infinite fusion multiplicities, the existence of a hierarchy of partly degenerate representations. We hope that this review will be enough for understanding the rest of the article, but we also recommend the reviews $[8,9]$ on $W_{n}$ algebras and [10] on conformal field theory. Then, we will solve the classical Toda equations on the disc (section 3 ). The resulting picture of the moduli spaces of D-branes will turn out to be qualitatively correct, as will be confirmed by the conformal bootstrap analysis (section 4). There, the analysis of the differential equations obeyed by certain two-point functions will result in explicit expressions for the one-point functions, which characterize how D-branes couple to bulk operators. The calculation of annulus partition functions will also provide some information on the boundary sector. The relation between the classical and bootstrap analyses will be made precise thanks to the light asymptotic limit (section 5), which will also allow us to predict some correlation functions which are at present out of reach of the bootstrap analysis. The conclusion (section 6) will summarize the main results and remaining puzzles. Then come two appendices, which are devoted to interesting but peripheral topics: appendix A to the minisuperspace limit, which will turn out to lead to the determination of the generating function of the Bäcklund transformation which relates $s \ell_{3}$ Toda classical mechanics to a free system, and appendix B to the existence of boundary actions, which we will be able to predict or rule out based on the properties of the boundary conditions.

\section{$2 \quad W_{n}$ algebras and their representations}

The symmetry algebra of the $s \ell_{n}$ conformal Toda theory is the so-called $W_{n}$ algebra. The Virasoro algebra coincides with the $W_{2}$ algebra, and is a subalgebra of the $W_{n>2}$ algebra, so that $s \ell_{n}$ conformal Toda theory indeed has conformal symmetry. The spectrum of the theory decomposes into representations of the $W_{n}$ algebra, which we will therefore study.

The infinite-dimensional $W_{n}$ algebra is related to the finite-dimensional $s \ell_{n}$ algebra in a number of ways. For example, the Virasoro algebra can be obtained from the affine extension $\widehat{s \ell_{2}}$ of the $s \ell_{2}$ algebra by a quantum Hamiltonian reduction of the Drinfeld-Sokolov type. The $W_{n}$ algebra can similarly be obtained from $\widehat{s \ell_{n}}$. Moreover, a fully degenerate representation of $W_{n}$ can be associated to each pair of two highest-weight representations of $s \ell_{n}$. This is our motivation for reviewing the representations of $s \ell_{n}$ (more on this in [10]), as an introduction to the study of representations of $W_{n}$.

\subsection{Representation theory of $s \ell_{n}$ Lie algebras}

Representations of $s \ell_{n}$ are parametrized by vectors in an $n$-1-dimensional space spanned by the simple roots $e_{1} \cdots e_{n-1}$ whose scalar products $K_{i, j}=\left(e_{i}, e_{j}\right)$ form the Cartan matrix, whose only nonzero entries are $K_{i i}=2, K_{i, i-1}=K_{i, i+1}=-1$. The $\frac{1}{2} n(n-1)$ positive roots 
are the sums of any numbers of consecutive simple roots, in the $s \ell_{3}$ case they are $\{e>$ $0\}=\left\{e_{1}, e_{2}, e_{1}+e_{2}\right\}$. The fundamental weights are the vectors $\omega_{i}$ such that $\left(\omega_{i}, e_{j}\right)=\delta_{i j}$. The Weyl vector is

$$
\rho=\frac{1}{2} \sum_{e>0} e=\sum_{i} \omega_{i}
$$

and $\rho^{2} \equiv(\rho, \rho)=\frac{1}{12}(n-1) n(n+1)$. In the $s \ell_{3}$ case we have

$$
\left\{\begin{array}{l}
\omega_{1}=\frac{2}{3} e_{1}+\frac{1}{3} e_{2} \\
\omega_{2}=\frac{1}{3} e_{1}+\frac{2}{3} e_{2}
\end{array},\left\{\begin{array}{l}
e_{1}=2 \omega_{1}-\omega_{2} \\
e_{2}=2 \omega_{2}-\omega_{1}
\end{array}, K=\left(\begin{array}{cc}
2 & -1 \\
-1 & 2
\end{array}\right),\{e>0\}=\left\{e_{1}, e_{2}, \rho\right\} .\right.\right.
$$

The Weyl group, a finite group, acts on the root space while preserving the scalar product. In the case of $s \ell_{2}$ it is a $\mathbb{Z}_{2}$ group whose nontrivial element is the reflection $r(v)=-v$. In the case of $s \ell_{3}$ the Weyl group has six elements $\{1, r, s, r s, s r, r s r=s r s\}$ and can be identified with the group of permutations of the three elements $\left\{h_{i}\right\} \equiv\left\{\omega_{1}, \omega_{2}-\omega_{1},-\omega_{2}\right\}$ with the action

\begin{tabular}{|c|c|c|c|c|c|}
\hline 1 & $r$ & $s$ & $r s$ & $s r$ & $r s r$ \\
\hline$e_{1}$ & $\rho$ & $-e_{1}$ & $-\rho$ & $e_{2}$ & $-e_{2}$ \\
$e_{2}$ & $-e_{2}$ & $\rho$ & $e_{1}$ & $-\rho$ & $-e_{1}$ \\
$\rho$ & $e_{1}$ & $e_{2}$ & $-e_{2}$ & $-e_{1}$ & $-\rho$ \\
\hline$\omega_{1}$ & $\omega_{1}$ & $\omega_{2}-\omega_{1}$ & $-\omega_{2}$ & $\omega_{2}-\omega_{1}$ & $-\omega_{2}$ \\
$\omega_{2}-\omega_{1}$ & $-\omega_{2}$ & $\omega_{1}$ & $\omega_{1}$ & $-\omega_{2}$ & $\omega_{2}-\omega_{1}$ \\
$-\omega_{2}$ & $\omega_{2}-\omega_{1}$ & $-\omega_{2}$ & $\omega_{2}-\omega_{1}$ & $\omega_{1}$ & $\omega_{1}$ \\
\hline
\end{tabular}

In the general $s \ell_{n}$ case, the Weyl group is generated by the $n-1$ reflections $s_{i}$ such that $s_{i}\left(e_{j}\right)=e_{j}-K_{j i} e_{i}$. The signature of an element of the group is the function $\epsilon$ such that $\epsilon\left(s_{i}\right)=-1$ and $\epsilon\left(w w^{\prime}\right)=\epsilon(w) \epsilon\left(w^{\prime}\right)$.

To an integral dominant weight, that is a vector $\Omega=\sum_{i} \lambda_{i} \omega_{i} \in \sum_{i} \mathbb{N} \omega_{i}$, we can associate a finite-dimensional irreducible representation $R_{\Omega}$ of $s \ell_{n}$. The vector $\Omega$ is then called its highest weight. A finite number of weights $h \in H_{\Omega}$ such that $\Omega-h \in \sum_{i} \mathbb{N} e_{i}$ are associated to the representation. The weights are the eigenvalues of the generators of the Cartan subalgebra when acting on a basis of the representation, so that the number of weights, taking into account their possible integer multiplicities, is the dimension of the representation. For example, the fundamental representation of $s \ell_{n}$ has dimension $n$ and weights $H_{\omega_{1}}=\left\{h_{k}=\omega_{1}-\sum_{i=1}^{k} e_{i} \mid k=0 \cdots n-1\right\}$. The adjoint representation of $s \ell_{3}$ has dimension 8 and weights $H_{\rho}=\left\{ \pm e_{1}, \pm e_{2}, \pm \rho, 2 \cdot 0\right\}$ where the weight 0 appears with multiplicity 2. Multiplicities higher than one appear only in the cases $s \ell_{n \geq 3}$.

The character $\chi_{\Omega}(p)$ of a representation is defined as a function of a vector $p$ by

$$
\chi_{\Omega}(p) \equiv \sum_{h \in H_{\Omega}} e^{(h, p)} .
$$

Given the highest weight $\Omega$ of a representation, the other weights can be found thanks to the Weyl formula

$$
\chi_{\Omega}(p)=\frac{\sum_{w \in W} \epsilon(w) e^{(\rho+\Omega, w(p))}}{\sum_{w \in W} \epsilon(w) e^{(\rho, w(p))}},
$$


whose denominator can be rewritten as

$$
\sum_{w \in W} \epsilon(w) e^{(\rho, w(p))}=\prod_{e>0}\left(e^{\frac{1}{2}(e, p)}-e^{-\frac{1}{2}(e, p)}\right) .
$$

Characters behave nicely under tensor products of representations, thanks to the property

$$
R_{\Omega} \otimes R_{\Omega^{\prime}}=\sum_{\Omega^{\prime \prime}} m_{\Omega, \Omega^{\prime}}^{\Omega^{\prime \prime}} R_{\Omega^{\prime \prime}} \Rightarrow \chi_{\Omega}(p) \chi_{\Omega^{\prime}}(p)=\sum_{\Omega^{\prime \prime}} m_{\Omega, \Omega^{\prime}}^{\Omega^{\prime \prime}} \chi_{\Omega^{\prime \prime}}(p) .
$$

The hyperplanes $\{(e, p)=0\}_{e>0}$ divide the $p$-space into $n$ ! Weyl chambers, which are fundamental domains for the action of the Weyl group.

For $n \geq 3$, the algebra $s \ell_{n}$ has an order two automorphism, called the Dynkin diagram automorphism, which maps $R_{\Omega}$ to $R_{\Omega^{*}}$, where the conjugation $\Omega \rightarrow \Omega^{*}$ is the linear map characterized by $e_{i}^{*}=e_{n-i}$. This map is trivial in the case of $s \ell_{2}$. We will see that this automorphism induces an automorphism of the $W_{n \geq 3}$ algebra.

\subsection{Representation theory of $W_{n}$ algebras}

The algebra $W_{n}$ is generated by $n-1$ operators $W^{(2)}, W^{(3)}, \cdots W^{(n)}$, where $W^{(2)}=T$ is the stress-energy tensor. (See the review [8].) Let us explicitly write Zamolodchikov's $W_{3}$ algebra, where for simplicity we denote $W^{(3)}=W$ :

$$
\begin{aligned}
T(z) T(w)= & \frac{c / 2}{(z-w)^{4}}+\frac{2 T(w)}{(z-w)^{2}}+\frac{\partial T(w)}{z-w}+\mathcal{O}(1) \\
T(z) W(w)= & \frac{3 W(w)}{(z-w)^{2}}+\frac{\partial W(w)}{(z-w)}+\mathcal{O}(1) \\
W(z) W(w)= & \frac{c / 3}{(z-w)^{6}}+\frac{2 T(w)}{(z-w)^{4}}+\frac{\partial T(w)}{(z-w)^{3}}+\frac{1}{(z-w)^{2}}\left[2 \beta \Lambda(w)+\frac{3}{10} \partial^{2} T(w)\right] \\
& +\frac{1}{z-w}\left[\beta \partial \Lambda(w)+\frac{1}{15} \partial^{3} T(w)\right]+\mathcal{O}(1)
\end{aligned}
$$

where $\Lambda(w)=(T T)(w)-\frac{3}{10} \partial^{2} T(w)$ and $\beta=\frac{16}{22+5 c}$. The algebra depends on a central charge $c$, which we parametrize in terms of a real number $b$ as $c=(n-1)(1+n(n+1)(b+$ $\left.\left.b^{-1}\right)^{2}\right)$. The generators of the algebra can be decomposed into modes $W_{n}^{(s)}$ as $W^{(s)}(z)=$ $\sum_{n \in \mathbb{Z}} W_{n}^{(s)} z^{-n-s}$; there is a special notation $L_{n}$ for the modes of $T(z)=\sum_{n \in \mathbb{Z}} L_{n} z^{-n-2}$.

A representation of the $W_{n}$ algebra can be encoded in a vertex operator $V(z)$, and the action of the algebra is encoded in the operator product $W^{(s)}(z) V(w)=\sum_{n \in \mathbb{Z}} \frac{W_{n}^{(s)} V(w)}{(z-w)^{s-n}}$. A standard assumption in conformal field theory is that the spectrum is a sum of highestweight representations, generated by primary operators such that $W_{n>0}^{(s)} V(w)=0$ and $W_{0}^{(s)} V(w)=q^{(s)} V(w)$. The product of a generator $W^{(s)}$ with a primary operator therefore contains a finite number of singular terms. In the $W_{3}$ case a primary operator $V(w)$ obeys

$$
\begin{aligned}
T(z) V(w) & =\frac{\Delta V(w)}{(z-w)^{2}}+\frac{\partial V(w)}{z-w}+\mathcal{O}(1), \\
W(z) V(w) & =\frac{q V(w)}{(z-w)^{3}}+\frac{W_{-1} V(w)}{(z-w)^{2}}+\frac{W_{-2} V(w)}{z-w}+\mathcal{O}(1),
\end{aligned}
$$


where we denote $\Delta=q^{(2)}$ the conformal dimension and $q=q^{(3)}$ the $W$-charge, and we use the identification of $L_{-1}$ with the generator of translations $\partial$, which is another standard assumption in conformal field theory. All operators of interest are assumed to be linear combinations of operators of the type $D V(w)=\left(\prod_{i=1}^{N} W_{-n_{i}}^{\left(s_{i}\right)}\right) V(w)$ where $V(w)$ is primary and $n_{i}>0, N \geq 0$. A descendent operator of level $L>0$ is a linear combination of such operators with $\sum_{i} n_{i}=L$. A descendent which is itself primary is called a null vector.

Representations are classified according to their numbers of algebraically independent null vectors. Each such null vector implies the existence of a relation between the $n-1$ charges $q^{(s)}$; so that the presence of $n-1$ null vectors would fully determine all charges as functions of the central charge $c$, and further null vectors would constrain $c$ itself. We work for generic values of $c$ and therefore consider only representations with a number $0 \leq k \leq n-1$ of algebraically independent null vectors. Such representations are called nondegenerate or continuous if $k=0$, simply degenerate if $k=1$, doubly degenerate if $k=2$, up to fully degenerate if $k=n-1$. Now the existence of $k$ algebraically independent null vectors implies the existence of $\frac{1}{2} k(k+1)$ linearly independent null vectors, and therefore $\frac{1}{2} k(k+1)$ null vector equations for correlation functions involving the corresponding primary operators. In particular, in the $s \ell_{3}$ case, each simply degenerate operator comes with one null-vector equation, whereas each fully degenerate operator comes with 3 null-vector equations.

A primary operator is in principle characterized by the corresponding $W_{0}^{(s)}$ eigenvalues $q^{(s)}$, but it is convenient to introduce a redundant parametrization of these eigenvalues and to label operators by an $(n-1)$-dimensional vector $\alpha$ called the momentum,

$$
\alpha=\sum_{i} \alpha_{i} \omega_{i} \quad \text { so that } \quad \alpha_{i}=\left(e_{i}, \alpha\right)
$$

The corresponding conformal dimension is supposed to be

$$
q_{\alpha}^{(2)}=\Delta_{\alpha}=\frac{1}{2}(\alpha, 2 Q-\alpha),
$$

where we introduce the vector

$$
Q=\left(b+b^{-1}\right) \rho .
$$

In the case of the algebra $W_{3}$ we also have

$$
q_{\alpha}^{(3)}=q_{\alpha}=\frac{i}{27}\left(\alpha_{1}-\alpha_{2}\right)\left(2 \alpha_{1}+\alpha_{2}-3 b-3 b^{-1}\right)\left(\alpha_{1}+2 \alpha_{2}-3 b-3 b^{-1}\right) .
$$

In general, $q^{(s)}$ is Weyl-invariant and homogeneous of degree $s$ as a function of $\alpha-Q$. The representations which appear in the spectrum of $s \ell_{3}$ conformal Toda theory have momenta

$$
\alpha \in Q+i\left(\mathbb{R} \omega_{1}+\mathbb{R} \omega_{2}\right),
$$

so that $\Delta_{\alpha}$ and $q_{\alpha}$ are real numbers. Under a Weyl transformation of the momentum, an operator $V_{\alpha}(z)$ is supposed to behave as

$$
V_{\alpha}(z)=R_{w}(\alpha) V_{Q+w(\alpha-Q)}(z)
$$


for some reflection coefficients $R_{w}(\alpha)$. On the other hand, the conjugation of $\alpha$ does not leave the charges invariant, but they transform according to $q_{\alpha^{*}}^{(s)}=(-1)^{s} q_{\alpha}^{(s)}$, because $\alpha^{*}$ is related to $2 Q-\alpha$ by a Weyl transformation. The conjugation of $\alpha$ therefore corresponds to the automorphism $W^{(s)} \rightarrow(-1)^{s} W^{(s)}$ of the algebra $W_{n}$. (This assumes that the charges $W^{(s \geq 3)}$ are defined so that they are primary operators of dimensions $s$ with respect to $T(z)$; other definitions are in principle possible.)

The character of a representation $R_{\alpha}$ of the $W_{n}$ algebra is defined by

$$
\xi_{\alpha}(\tau) \equiv \operatorname{Tr}_{R_{\alpha}} e^{2 i \pi \tau\left(L_{0}-\frac{c}{24}\right)} .
$$

This is easily computed in the case of a continuous representation, with the result

$$
\xi_{\alpha}(\tau)=\frac{e^{-i \pi \tau(Q-\alpha)^{2}}}{\eta\left(e^{2 i \pi \tau}\right)^{n-1}},
$$

where $\eta$ is the Dedekind eta function. Let us now consider the case of a fully degenerate representation of the $W_{n}$ algebra. Such a representation $R_{-b \Omega^{+}-b^{-1} \Omega^{-}}$, which is generated by the primary operator $V_{-b \Omega^{+}-b^{-1} \Omega^{-}}$, can be associated to any pair $\left(\Omega^{+}, \Omega^{-}\right)$of integral dominant weights of $s \ell_{n}$. The character $R_{-b \Omega^{+}-b^{-1} \Omega^{-}}$is a sum over the Weyl group [11],

$$
\xi_{-b \Omega^{+}-b^{-1} \Omega^{-}}(\tau)=\frac{\sum_{w \in W} \epsilon(w) e^{-i \pi \tau\left(b\left(\rho+\Omega^{+}\right)+b^{-1} w\left(\rho+\Omega^{-}\right)\right)^{2}}}{\eta\left(e^{2 i \pi \tau}\right)^{n-1}} .
$$

Now we observe that this degenerate character can be expressed in terms of the characters $\chi_{\Omega^{ \pm}}$of the two representations of $s \ell_{n}$ of highest weights $\Omega^{ \pm}$,

$$
\begin{aligned}
\xi_{-b \Omega^{+}-b^{-1} \Omega^{-}}(\tau)= & \frac{\sqrt{n}}{n !} \int d^{(n-1)} p \frac{e^{-\frac{2 i \pi}{\tau} \frac{1}{2} p^{2}}}{\eta\left(e^{-\frac{2 i \pi}{\tau}}\right)^{n-1}} \\
& \times \prod_{ \pm}\left[\chi_{\Omega^{ \pm}}\left(2 \pi b^{ \pm 1} p\right) \prod_{e>0}\left(e^{\frac{1}{2}\left(e, 2 \pi b^{ \pm 1} p\right)}-e^{-\frac{1}{2}\left(e, 2 \pi b^{ \pm 1} p\right)}\right)\right],
\end{aligned}
$$

where the integration measure is defined as $d^{(n-1)} p=\prod_{i=1}^{n-1} d p_{i}$ with $p=\sum_{i} p_{i} e_{i}$, and we used the Weyl formula (2.5).

Notice that the $W_{n}$ characters $\xi_{\alpha}(\tau)$ keep track of the conformal dimensions ( $L_{0}$ eigenvalues) of states, and not of their charges $q^{(s>2)}$. So if $n>2$ they contain much less information than the $s \ell_{n}$ characters $\chi_{\Omega}(p)$, which depend on a vector $p$ and not on a single number $\tau$. This will make the modular bootstrap analysis less powerful in theories with $W_{n>2}$ symmetries than in theories with just the Virasoro symmetry.

\subsection{Fusion multiplicity}

We will now comment on the fusion product of $W_{n}$ representations. The fusion product is a generalisation to vertex operator algebras of the tensor product of representations of Lie algebras. So we first comment on the tensor product of $s \ell_{n}$ representations. We consider generic representations, which are not necessarily finite-dimensional, and even do not necessarily have a highest weight. 
The algebra $s \ell_{n}$ can be represented in terms of differential operators acting on functions of $\frac{1}{2} n(n-1)$ "isospin" variables. (This is also the number of the creation operators, the operators which generate the highest-weight representations from their highest-weight states.) For example, $s \ell_{2}$ is represented by the operators $D^{-}=\frac{\partial}{\partial x}, D^{3}=x \frac{\partial}{\partial x}-j, D^{+}=x^{2} \frac{\partial}{\partial x}-2 j x$ acting on functions of one isospin variable $x$, where the number $j$ is the spin of the representation. States in a representation of $s \ell_{n}$ with spin $j$ can be represented as functions $\Psi^{j}(x)$, where the spin $j$ is a vector with $n-1$ components, and the isospin $x$ is a vector with $\frac{1}{2} n(n-1)$ components. We wish to analyse the possible appearances of a representation $R_{j_{3}}$ in the tensor product $R_{j_{1}} \otimes R_{j_{2}}$. Such an appearance implies the existence of a nonzero invariant vector in $R_{j_{1}} \otimes R_{j_{2}} \otimes R_{j_{3}}^{*}$, where $R_{j_{3}}^{*}$ is the contragredient representation. In the representation of $s \ell_{n}$ in terms of differential operators, an invariant vector in $R_{j_{1}} \otimes R_{j_{2}} \otimes R_{j_{3}}^{*}$ is represented as a function $\Phi\left(x_{1}, x_{2}, x_{3}\right)$ of three isospin vectors, subject to $\left(\operatorname{dim} s \ell_{n}\right)=n^{2}-1$ equations. If the representations are generic and no more assumptions are made, solutions $\Phi\left(x_{1}, x_{2}, x_{3}\right)$ come with the number of parameters

$$
d_{n}=3 \frac{n(n-1)}{2}-\left(n^{2}-1\right)=\frac{1}{2}(n-1)(n-2) .
$$

If $n>2$ then $d_{n}>0$ which implies that $R_{j_{3}}$ can appear an infinite number of times in $R_{j_{1}} \otimes R_{j_{2}}$. If however one of the three representations $R_{j_{1}}, R_{j_{2}}$ or $R_{j_{3}}$ is not generic, then extra equations on $\Phi\left(x_{1}, x_{2}, x_{3}\right)$ can follow, and the number of parameters may become lower. If the number of parameters is zero, as happens if $n=2$ or one of the involved representations has a highest weight state, then multiplicities must be finite.

A similar counting of variables, and similar conclusions on fusion multiplicities, hold in the case of the fusion product of $W_{n}$ representations. This is a consequence of the conformal Ward identities for the three-point correlation functions $\left\langle\prod_{i=1}^{3} V_{\alpha_{i}}\left(z_{i}\right)\right\rangle$ where the momenta $\alpha_{i}$ label $W_{n}$ representations; such correlation functions are analogous to the invariants $\Phi\left(x_{1}, x_{2}, x_{3}\right)$ of our $s \ell_{n}$ reasoning (although the positions $z_{i}$ are not analogous to the isospins $x_{i}$ ). The fusion multiplicity is the minimum number of correlation functions of descendent operators $\left\langle\prod_{i=1}^{3} D_{i} V_{\alpha_{i}}\left(z_{i}\right)\right\rangle$ in terms of which all other such correlation functions can be linearly expressed using the Ward identities. Such identities are obtained by inserting the identity $\oint_{\infty} \varphi_{s}(z) W^{(s)}(z)=0$ in a correlation function, where $\oint_{\infty}$ denotes the integration along a contour which encloses all the positions $z_{i}$ of the operators, $\varphi_{s}(z)$ is meromorphic with possible poles at $z=z_{i}$, and at infinity $\left|\varphi_{s}(z)\right| \leq|z|^{2 s-2}$. (We assume $W^{(s)}(z) \underset{z \rightarrow \infty}{\sim} z^{-2 s}$, which follows from the $W^{(s)}$-symmetry of the vacuum.) Local Ward identities are obtained for functions $\varphi_{s}(z)$ which do have poles; the case $\varphi_{s}(z)=\left(z-z_{i}\right)^{-k}$ (with $k \in \mathbb{N}$ ) yields the expression of a correlation function involving $W_{1-s-k}^{(s)} V_{\alpha_{i}}\left(z_{i}\right)$ in terms of correlation functions with descendents of the type $W_{-p}^{(s)} V_{\alpha_{i}}\left(z_{i}\right)$ with $1 \leq p \leq s-1$, as can be seen from the operator product $W^{(s)}(z) V_{\alpha}(w)$. (See eq. (2.12) for the case $s=3$.) In the theory with $W_{n}$ symmetry there are $\frac{1}{2} n(n-1)$ modes of the type $W_{-p}^{(s)}$ with $2 \leq s \leq n, 1 \leq p \leq s-1$, for instance the three modes $L_{-1}, W_{-1}, W_{-2}$ in the case $n=3$, and these modes are analogous to the isospin variables $x$ of the $s \ell_{n}$ algebra. Global Ward identities are obtained for holomorphic functions $\varphi_{s}(z)$, that is polynomials of degrees at most $2 s-2$. The number of global Ward identities is therefore $\sum_{s=2}^{n}(2 s-1)=n^{2}-1$. 
Thus, the number of modes of the $W^{(s)}$ symmetry generators which cannot be eliminated from the correlation functions of the type $\left\langle\prod_{i=1}^{3} D_{i} V_{\alpha_{i}}\left(z_{i}\right)\right\rangle$ using the Ward identities is $d_{n}$ (2.23). For example, $d_{3}=1$ means that in a theory with $W_{3}$ symmetry all three-point functions can be expressed in terms of the correlation functions $\left\langle V_{\alpha_{1}}\left(z_{1}\right) V_{\alpha_{2}}\left(z_{2}\right)\left(W_{-1}\right)^{k} V_{\alpha_{3}}\left(z_{3}\right)\right\rangle$ where $V_{\alpha_{i}}$ are primary operators and $k \in \mathbb{N}$. (Instead of $W_{-1}$ we may have written $W_{-2}$, but not $L_{-1}$, because the three global Ward identities from $T(z)$ close among themselves and can be solved.) Notice that a similar reasoning can be used to predict the number $E$ of independent differential equations obeyed by an $N$ point function of primary operators, some of which may be degenerate and involve a total number of $V$ linearly independent null vectors. We find $E=n^{2}-4-N\left(\frac{n(n-1)}{2}-1\right)+V$, where we subtract the three equations from global conformal symmetry, as well as the contribution of the $L_{-1}$ generator as it is identified with a derivative. Such a counting of differential equations has previously been used in [12].

To conclude, infinite fusion multiplicities must appear in all theories with a $W_{n \geq 3}$ symmetry as soon as continuous representations are involved, which will be the case in conformal Toda theories.

\subsection{Lagrangian formulation}

Conformal $s \ell_{n}$ Toda theory on a Riemann surface without boundary has a Lagrangian formulation. The dynamical fields of the theory form a vector with $n-1$ components

$$
\phi=\sum_{i} \phi_{i} e_{i} \quad \text { so that } \quad \phi_{i}=\left(\omega_{i}, \phi\right)
$$

and the Lagrangian is

$$
L_{n}=\frac{1}{2 \pi}(\partial \phi, \bar{\partial} \phi)+\mu \sum_{i=1}^{n-1} e^{b\left(e_{i}, \phi\right)},
$$

where $\mu$ is the bulk cosmological constant, and the derivatives with respect to the complex coordinates $z, \bar{z}$ are related to derivatives with respect to the real coordinates $x=\Re z, y=$ $\Im z$ by $\partial=\frac{1}{2}\left(\frac{\partial}{\partial x}-i \frac{\partial}{\partial y}\right), \bar{\partial}=\frac{1}{2}\left(\frac{\partial}{\partial x}+i \frac{\partial}{\partial y}\right)$. After the rescaling $\phi \rightarrow b^{-1} \phi$, the classical equations of motion are

$$
\partial \bar{\partial} \phi_{i}=\pi b^{2} \mu e^{\left(e_{i}, \phi\right)} .
$$

The Lagrangian formulation permits the calculation of certain particular correlation functions, and of general correlation functions in certain limits, but not of general correlation function [1, 13]. For our purposes, we will only make use of the classical equations of motion, and not of functional integrals involving the action $S=\int L_{n}$. We will actually solve the equations of motion in section 3 .

The $W_{n}$ symmetry of $s \ell_{n}$ Toda theory manifests itself by the existence of charges $W^{(2)}=T, W^{(3)} \cdots W^{(n)}$ which are classically conserved in the sense that $\bar{\partial} W^{(s)}=0$. In the case of Liouville theory, this is

$$
T=-(\partial \phi)^{2}+\partial^{2} \phi
$$


In the case of $s \ell_{3}$ Toda theory, $W^{(3)}=W$ has the ambiguity $W \rightarrow W-\xi \partial T$ for $\xi$ an arbitrary number, which we lift by assuming $W\left(\phi^{*}\right)=-W(\phi)$, where $\phi \rightarrow \phi^{*}$ is the Dynkin diagram automorphism $\phi_{1} \leftrightarrow \phi_{2}$. We then have

$$
\begin{aligned}
T & =-\frac{1}{2}(\partial \phi, \partial \phi)+\left(\rho, \partial^{2} \phi\right)=-\partial \phi_{1}^{2}-\partial \phi_{2}^{2}+\partial \phi_{1} \partial \phi_{2}+\partial^{2} \phi_{1}+\partial^{2} \phi_{2}, \\
W & =\left(\partial^{3} \phi_{2}-2 \partial^{2} \phi_{2} \partial \phi_{2}-\partial^{2} \phi_{2} \partial \phi_{1}+2 \partial \phi_{2}^{2} \partial \phi_{1}\right)-\left(\phi_{1} \leftrightarrow \phi_{2}\right),
\end{aligned}
$$

where we neglect a possible normalization factor in the definition of $W$. Such classically conserved charges can alternatively be found as classical limits of the corresponding quantum symmetry generators of the $W_{3}$ algebra [5]. We use the same notation for the classical charge and the quantum generator; the context should clarify which one we are dealing with.

The momentum $\alpha$, which we used as a label for $W_{n}$ representations (see (2.14), (2.16)), has a simple interpretation in the Lagrangian formulation. Namely, the classical counterpart of the quantum operator $V_{\alpha}(z)$ is $e^{(\alpha, \phi(z))}$.

\section{Solutions of the Toda equations on a disc}

The classical $s \ell_{n}$ Toda equations on the Riemann sphere have been solved in [14], see also [15]. We will now look for solutions on the disc, which are solutions on the sphere respecting certain boundary conditions. We will only consider maximally symmetric boundary conditions, that is conditions of the type $\bar{W}^{(s)}=f\left(\left\{W^{\left(s^{\prime}\right)}\right\}\right)$ where $f$ is an automorphism of the $W_{n}$ algebra. The known automorphisms are the identity, and in the $W_{n \geq 3}$ algebra the automorphism $W^{(s)} \rightarrow(-1)^{s} W^{(s)}$. There will therefore be two possible types of boundary conditions if $n \geq 3$, and only one if $n=2$. We will study the cases of the $W_{2}$ and $W_{3}$ algebras. The sphere will be identified with the complex plane and parametrized by the coordinates $z, \bar{z}$, and the disc will be identified with the upper half-plane $\{\Im z>0\}$.

In this section, we will study the solutions of the Toda equations and in particular their invariants, which we call the boundary parameters. The question whether our boundary conditions follow from boundary actions is postponed to appendix B. We will consider the classical Toda equations (2.26) with the value

$$
\mu=-\frac{1}{\pi b^{2}}
$$

for the cosmological constant. The choice of a negative value for $\mu$ will allow real, globally defined, regular solutions to exist.

\subsection{Case of Liouville theory}

In order to solve the Liouville equation $\partial \bar{\partial} \phi=-e^{2 \phi}$ together with the boundary condition $T=\bar{T}$ where $T=-(\partial \phi)^{2}+\partial^{2} \phi$, we introduce the variable $X=e^{-\phi}$ which is such that $T=-\frac{\partial^{2} X}{X}$ and the Liouville equation amounts to $\Delta_{2}(X) \equiv X \partial \bar{\partial} X-\partial X \bar{\partial} X=1$. The solutions of this equation are of the form

$$
X=\sum_{i=1}^{2} a_{i}(z) b_{i}(\bar{z}), \quad \operatorname{Wr}\left[a_{1}, a_{2}\right]=\mathrm{Wr}\left[b_{1}, b_{2}\right]=1,
$$


where $\operatorname{Wr}\left[a_{1}, a_{2}\right]=a_{1} a_{2}^{\prime}-a_{2} a_{1}^{\prime}$ is the Wronskian. (By setting $\operatorname{Wr}\left[a_{1}, a_{2}\right]=\operatorname{Wr}\left[b_{1}, b_{2}\right]=1$ we have eliminated the ambiguity $a_{i} \rightarrow \xi a_{i}, b_{i} \rightarrow \xi^{-1} b_{i}$.) The stress-energy tensor $T$ associated with such a solution is $T=-\frac{a_{1}^{\prime \prime}}{a_{1}}=-\frac{a_{2}^{\prime \prime}}{a_{2}}$. The condition $T=\bar{T}$ is solved by assuming

$$
a_{i}(z)=\sum_{j=1}^{2} N_{i j} b_{j}(z), \quad \operatorname{det} N=1
$$

The condition that $X$ be real and positive will now be solved by assuming that $b(z)=\bar{b}(z)$ is real, and that the constant matrix $N$ is Hermitian and positive. To summarize, our solutions are

$$
X=\sum_{i, j=1}^{2} \overline{b_{i}(z)} N_{i j} b_{j}(z), \quad \operatorname{Wr}\left[b_{1}, b_{2}\right]=1, \operatorname{det} N=1, N>0 .
$$

There remain some ambiguities in the solutions, because different choices for $N_{i j}, b_{i}(z)$ can lead to the same $X$. In particular, the following action of $S L(2, \mathbb{R})$ leaves $X$ invariant:

$$
\left\{\begin{array}{l}
b \rightarrow \Lambda^{-1} b \\
N \rightarrow \Lambda^{T} N \Lambda
\end{array}, \quad \Lambda \in S L(2, \mathbb{R})\right.
$$

where $\Lambda^{T}$ denotes the transpose of the matrix $\Lambda$.

Let us define a boundary parameter $\lambda_{L}$ associated to a given solution. We assume this parameter to be a $z$-independent function of the solution $X$. Independence from $z$ implies being a function of the matrix $N$. Being a function of $X$ implies being invariant under the action $(3.5)$ of $S L(2, \mathbb{R})$. The only such invariant function of $N$ is

$$
\lambda_{L} \equiv \frac{1}{2 i} \operatorname{Tr} N P, \quad P \equiv\left(\begin{array}{cc}
0 & -1 \\
1 & 0
\end{array}\right) .
$$

Notice that the matrix $P$ obeys $\forall \Lambda \in S L(2, \mathbb{R}), \Lambda P \Lambda^{T}=P$. The role of $\lambda_{L}$ as a boundary parameter can be demonstrated by rewriting the boundary condition in terms of the field $X=e^{-\phi}$. At the boundary $z=\bar{z}$ we find:

$$
(\partial-\bar{\partial}) X=2 i \lambda_{L}, \quad(\partial-\bar{\partial}) \phi=-2 i \lambda_{L} e^{\phi} .
$$

This implies that the boundary conditions could be derived by adding a boundary term $\int L_{2}^{\text {bdy }}$ to the action, with

$$
L_{2}^{\mathrm{bdy}}=\lambda_{L} e^{\phi},
$$

and $\lambda_{L}$ would be the boundary cosmological constant.

We conclude this subsection with a remark. Given the solution of the Liouville equation, it is easy to write the Bäcklund transformation from Liouville theory to a free field theory. The free field can be defined as $\psi=-\log \frac{\sum_{i} u_{i} a_{i}(z)}{\sum_{i} v_{i} b_{i}(\bar{z})}$ where $\left(u_{1}, u_{2}\right)$ and $\left(v_{1}, v_{2}\right)$ are constant vectors, and the stress-energy tensors are $T=-(\partial \psi)^{2}+\partial^{2} \psi, \bar{T}=-(\bar{\partial} \psi)^{2}-\bar{\partial}^{2} \psi$. The field $\psi$ obeys the free equations of motion $\partial \bar{\partial} \psi=0$, as well as Dirichlet boundary conditions $(\partial+\bar{\partial}) \psi=0$, and the value of $\psi$ at the boundary is related to $\lambda_{L}$. 


\subsection{Case of $s \ell_{3}$ Toda theory}

Let us solve the $s \ell_{3}$ Toda equations $\left\{\begin{array}{l}\partial \bar{\partial} \phi_{1}=-e^{2 \phi_{1}-\phi_{2}} \\ \partial \bar{\partial} \phi_{2}=-e^{2 \phi_{2}-\phi_{1}}\end{array}\right.$. In terms of $X_{i}=e^{-\phi_{i}}$, the $s \ell_{3}$ Toda equations amount to $\left\{\begin{array}{l}\Delta_{2}\left(X_{1}\right)=X_{2} \\ \Delta_{2}\left(X_{2}\right)=X_{1}\end{array}\right.$, where $\Delta_{2}(X)$ was defined in the previous subsection, and is such that $\Delta_{2}\left(\Delta_{2}(X)\right)=X \operatorname{det}\left(\begin{array}{ccc}X & \partial X X & \partial \partial X \\ \bar{\partial} X & \partial \bar{\partial} X & \partial \partial \bar{\partial} X \\ \partial \bar{\partial} X & \partial \bar{\partial} \bar{\partial} X & \partial \partial \partial \bar{\partial} X\end{array}\right)$. The solutions of the $s \ell_{3}$ Toda equations are

$$
\left\{\begin{array}{l}
X_{1}=\sum_{i=1}^{3} a_{i}(z) b_{i}(\bar{z}) \\
X_{2}=\sum_{i<j} \operatorname{Wr}\left[a_{i}, a_{j}\right](z) \mathrm{Wr}\left[b_{i}, b_{j}\right](\bar{z})
\end{array}, \quad \operatorname{Wr}\left[a_{1}, a_{2}, a_{3}\right]=\mathrm{Wr}\left[b_{1}, b_{2}, b_{3}\right]=1,\right.
$$

where $\operatorname{Wr}\left[a_{1}, a_{2}, a_{3}\right]=\epsilon_{i j k} a_{i} a_{j}^{\prime} a_{k}^{\prime \prime}$ is the cubic Wronskian. In this solution, the Dynkin diagram automorphism $\phi_{1} \leftrightarrow \phi_{2}$ manifests itself as $\left\{\begin{array}{l}a_{i} \leftrightarrow \frac{1}{2} \epsilon_{i j k} \mathrm{Wr}\left[a_{j}, a_{k}\right] \\ b_{i} \leftrightarrow \frac{1}{2} \epsilon_{i j k} \mathrm{Wr}\left[b_{j}, b_{k}\right]\end{array}\right.$. In these formulas we used the fully antisymmetric tensor $\epsilon_{i j k}$ such that $\epsilon_{123}=1$.

Let us rewrite the symmetry charges $T(2.28)$ and $W(2.29)$ in terms of the variables $X_{1}, X_{2}$ or $a_{i}, b_{j}$ :

$$
\begin{aligned}
T & =-\frac{\partial^{2} X_{1}}{X_{1}}-\frac{\partial^{2} X_{2}}{X_{2}}+\frac{\partial X_{1}}{X_{1}} \frac{\partial X_{2}}{X_{2}}=\frac{\partial^{3} X_{1} \bar{\partial} X_{1}-X_{1} \partial^{3} \bar{\partial} X_{1}}{X_{2}}=\frac{a_{3} a_{1}^{\prime \prime \prime}-a_{1} a_{3}^{\prime \prime \prime}}{\operatorname{Wr}\left[a_{1}, a_{3}\right]}, \\
W & =T\left(\frac{\partial X_{2}}{X_{2}}-\frac{\partial X_{1}}{X_{1}}\right)-\frac{\partial^{3} X_{1}}{X_{1}}+\frac{\partial^{3} X_{2}}{X_{2}}=-T^{\prime}+2 \frac{a_{1}^{\prime} a_{3}^{\prime \prime \prime}-a_{3}^{\prime} a_{1}^{\prime \prime \prime}}{\operatorname{Wr}\left[a_{1}, a_{3}\right]}
\end{aligned}
$$

where we could use any pair of functions $a_{i}$ instead of $\left(a_{1}, a_{3}\right)$, and the result would not change due to the identity $\mathrm{Wr}\left[a_{1}, a_{2}, a_{3}\right]^{\prime}=0$. The antiholomorphic charges $\bar{T}, \bar{W}$ are similarly written in terms of $b_{i}$.

We now consider $s \ell_{3}$ Toda theory on the half-plane, and the possible boundary conditions on the real line. We must impose $T=\bar{T}$ for conformal symmetry to be preserved. For the spin 3 current $W$ we have the two choices $W= \pm \bar{W}$, where the minus sign corresponds to using the nontrivial automorphism of the $W_{3}$ algebra.

\subsection{Boundary condition $W-\bar{W}=0$}

Now that we wrote the solutions of the bulk equations of motion in terms of the functions $a_{i}, b_{i}$, let us write boundary conditions for these functions. The conditions $T=\bar{T}, W=\bar{W}$ are obeyed provided we assume

$$
a_{i}=\sum_{j=1}^{3} N_{i j} b_{j}, \quad \operatorname{det} N=1
$$

This implies $\epsilon_{i j k} \mathrm{Wr}\left[a_{j}, a_{k}\right]=\left(N^{-1 T}\right)_{i i^{\prime}} \epsilon_{i^{\prime} j^{\prime} k^{\prime}} \mathrm{Wr}\left[b_{j^{\prime}}, b_{k^{\prime}}\right]$, where $N^{-1 T}$ is the inverse of the transpose of $N$. Furthermore, in order for $X_{1}, X_{2}$ to be positive, we assume $b_{i}=\bar{b}_{i}$ and that $N$ is a positive Hermitian matrix. To summarize,

$$
\left\{\begin{array}{l}
X_{1}=\sum_{i, j=1}^{3} \overline{b_{i}(z)} N_{i j} b_{j}(z) \\
X_{2}=\sum_{i, j=1}^{3} \overline{w_{i}(z)}\left(N^{-1 T}\right)_{i j} w_{j}(z)
\end{array}, \quad \operatorname{Wr}\left[b_{1}, b_{2}, b_{3}\right]=1, \operatorname{det} N=1, N>0,\right.
$$


where we introduced the notation $w_{i}=\epsilon_{i j k} b_{j} b_{k}^{\prime}$ for the quadratic Wronskians, whose quadratic Wronskians are themselves $\operatorname{Wr}\left[w_{i}, w_{j}\right]=\epsilon_{i j k} b_{k}$.

As in the case of Liouville theory, our parametrization of the solutions is ambiguous, because $X_{1}, X_{2}$ are invariant under the action of an $\mathrm{SL}(3, \mathbb{R})$ symmetry group, namely

$$
\left\{\begin{array}{l}
b \rightarrow \Lambda^{-1} b \\
w \rightarrow \Lambda^{T} w \\
N \rightarrow \Lambda^{T} N \Lambda
\end{array}, \quad \Lambda \in \mathrm{SL}(3, \mathbb{R}) .\right.
$$

The boundary parameters are the $\operatorname{SL}(3, \mathbb{R})$-invariant functions of $N$. Such invariants can be constructed as $\operatorname{Tr}\left(N^{T} N^{-1}\right)^{m}, m=1,2 \cdots$. Given $\operatorname{det} N=1$, all such invariants are functions of

$$
\lambda_{0} \equiv \operatorname{det} \frac{1}{2}\left(N+N^{T}\right) .
$$

In the $s \ell_{n}$ case the number of invariants is the integer part of $\frac{n}{2}$.

We may wish to express the boundary conditions in terms of the fields $X_{i}=e^{-\phi_{i}}$. To this end, we may compute at the boundary

$$
\left\{\begin{array}{rl}
(\partial-\bar{\partial}) X_{1} & =\frac{1}{2} \epsilon_{i j k} N_{i j} w_{k} \\
(\partial-\bar{\partial}) X_{2} & =\frac{1}{2} \epsilon_{i j k} N_{i j}^{-1 T} b_{k}
\end{array} .\right.
$$

The right hand sides of these expressions are in general not functions of $X_{1}, X_{2}$. There is an exception in the special case when $N^{T}=N$, which corresponds to the free boundary conditions $(\partial-\bar{\partial}) X_{i}=0$, in which case the boundary parameter is $\lambda_{0}=1$. Another exception occurs when $N_{i j}=U_{i} U_{j}+\epsilon_{i j k} A_{k}$, with $A_{i} U_{i}=1$ so that $\operatorname{det} N=1$. (We drop the assumption that $N$ be hermitian.) In this case the boundary parameter is $\lambda_{0}=-1$. Noticing $N^{-1 T}=A_{i} A_{j}+\epsilon_{i j k} U_{k}$, we find $\left\{\begin{array}{l}(\partial-\bar{\partial}) X_{1}=A_{k} w_{k}=\sqrt{X_{2}} \\ (\partial-\bar{\partial}) X_{2}=U_{k} b_{k}=\sqrt{X_{1}}\end{array}\right.$. Such boundary conditions derive from the boundary Lagrangian

$$
L_{3}^{\mathrm{bdy}}=\frac{1}{2 i}\left(e^{\phi_{1}-\frac{1}{2} \phi_{2}}+e^{\phi_{2}-\frac{1}{2} \phi_{1}}\right) .
$$

One may be tempted to generalize this Lagrangian into $L_{3}^{\text {bdy }}=\nu_{1} e^{\phi_{1}-\frac{1}{2} \phi_{2}}+\nu_{2} e^{\phi_{2}-\frac{1}{2} \phi_{1}}$, which would depend on two boundary parameters $\nu_{1}, \nu_{2}$. However, it turns out that for general values of $\nu_{1}, \nu_{2}$ the $W_{3}$ symmetry would then be broken, in the sense that the boundary condition $W=\bar{W}$ would not be obeyed. Only the values of $\nu_{1}, \nu_{2}$ which we wrote in eq. (3.17) are therefore permitted.

\subsection{Boundary condition $W+\bar{W}=0$}

Boundary conditions for the functions $a_{i}, b_{i}$ which imply $W+\bar{W}=0$ are now

$$
a_{i}=\sum_{j=1}^{3} N_{i j} w_{j}, \quad \operatorname{det} N=1 .
$$


This can be deduced from the case $W-\bar{W}=0$ by using the Dynkin diagram automorphism, which exchanges the functions $b_{i}$ with their Wronskians $w_{i}=\epsilon_{i j k} b_{j} b_{k}^{\prime}$. It is however not clear how to guarantee the positivity of $X_{1}, X_{2}$. We refrain from making further assumptions on the matrix $N$, and we write the solutions of the classical Toda equations as

$$
\left\{\begin{array}{l}
X_{1}=\sum_{i, j=1}^{3} b_{i}(\bar{z}) N_{i j} w_{j}(z) \\
X_{2}=\sum_{i, j=1}^{3} w_{i}(\bar{z}) N_{i j}^{-1 T} b_{j}(z)
\end{array}, \quad \operatorname{Wr}\left[b_{1}, b_{2}, b_{3}\right]=1, \operatorname{det} N=1 .\right.
$$

As we do not impose reality conditions on $b_{i}$ and $N$ we find that $X_{1}, X_{2}$ are invariant under the action of an $\operatorname{SL}(3, \mathbb{C})$ group of symmetries, instead of an $\operatorname{SL}(3, \mathbb{R})$ group in the case $W-\bar{W}=0$ :

$$
\left\{\begin{array}{l}
b \rightarrow \Lambda^{-1} b \\
w \rightarrow \Lambda^{T} w \\
N \rightarrow \Lambda^{T} N \Lambda^{-1 T}
\end{array} \quad, \quad \Lambda \in \mathrm{SL}(3, \mathbb{C}) .\right.
$$

The group acts by conjugation on the matrix $N$, and there are two invariants, which we interpret as boundary parameters:

$$
\lambda_{1}=\operatorname{Tr} N, \quad \lambda_{2}=\operatorname{Tr} N^{-1} .
$$

In the $s \ell_{n}$ case the number of invariants is of course $n-1$. This corresponds to the number of conserved charges $W^{(2)} \cdots W^{(n)}$. This already suggests that the boundary condition $W+\bar{W}=0$ realizes Cardy's ideas on the correspondence between representations of the symmetry algebra and boundary parameters. We will demonstrate this further in our conformal bootstrap analysis in section 4 .

An interesting case happens when the matrix $N$ obeys a second-order polynomial equation, that is when two of its eigenvalues coincide. Then we have $N^{-1}=u N+v I$ where $I$ is the identity matrix and $u, v$ are two complex numbers, and it follows from eq. (3.19) that $\phi_{1}-\phi_{2}$ obeys Dirichlet boundary conditions and $\phi_{1}+\phi_{2}$ obeys Neumann boundary conditions,

$$
\phi_{1}-\phi_{2}=c, \quad(\partial-\bar{\partial})\left(\phi_{1}+\phi_{2}\right)=0,
$$

where $c$ is an arbitrary constant. These conditions can be derived from the $s \ell_{3}$ Toda action with no boundary terms. Due to the Dirichlet condition, the spacetime interpretation of this case is a one-dimensional D-brane, whereas the other cases describe two-dimensional D-branes. The one-dimensional D-branes extend along the direction of the Weyl vector $\rho$, which is consistent with the existence of a linear dilaton in that direction. (This linear dilaton can be seen in the expression for the stress-energy tensor $T$ (2.28).)

We conclude this subsection with a remark. Given the solution of the Toda equations, it is easy to write the Bäcklund transformation from Toda field theory to a free field theory. The free fields can be defined as $\psi_{i}=-\log \frac{\sum_{j} u_{i j} a_{j}(z)}{\sum_{j} v_{i j} w_{j}(\bar{z})}$ where $u_{i j}$ and $v_{i j}$ are constant matrices. The free fields obey free equations of motion $\partial \bar{\partial} \psi_{i}=0$, and Dirichlet boundary conditions for $\psi_{i}$ imply relations of the type $a_{i}=\sum_{j} N_{i j} w_{j}$ (3.18) at the boundary. Thus, we can interpret the boundary parameters $\lambda_{1}, \lambda_{2}$ encoded in the matrix $N$ as the boundary values of the free fields. (See appendix A for more details on the Bäcklund transformation.) 


\section{Conformal bootstrap study of $s \ell_{3}$ Toda theory with $W+\bar{W}=0$}

The conformal bootstrap method is the systematic exploitation of symmetry and consistency constraints on correlation functions in two-dimensional conformal field theories [4]. We will apply this method to the correlation functions of $W_{3}$ primary operators $V_{\alpha}(z)$. Such operators are defined up to normalizations by their operator products (2.11) and (2.12) with the symmetry generators $T(z), W(z), \bar{T}(\bar{z}), \bar{W}(\bar{z})$. In order to define correlation functions on the upper half-plane, the properties of the boundary must be characterized. This involves first of all imposing boundary conditions for the symmetry generators $T(z), W(z)$. Moreover, we saw in section 3 that for each of the two boundary conditions $\left\{\begin{array}{l}T=\bar{T} \\ W= \pm \bar{W}\end{array}\right.$ there exist families of possible D-branes, parametrized by $\lambda_{0}$ or $\lambda_{1}, \lambda_{2}$. These parameters appeared in the classical analysis of the boundary conditions for the basic Toda fields $\phi_{i}$, but such fields are not present in the conformal bootstrap formalism. Nevertheless, the equivalents of $\lambda_{0}$ or $\lambda_{1}, \lambda_{2}$ will appear when we will parametrize the solutions of the conformal bootstrap equations; a given solution will be called a D-brane or boundary state.

In the case of Liouville theory there is only one possible boundary condition $T=\bar{T}$, and there exist two types of D-branes: the continuous D-branes [16, 17], with a continuous parameter, and the discrete D-branes [18], which are parametrized by two integers. These two types of D-branes are associated to the two types of representations of the Virasoro algebra: the continuous and discrete representations. By analogy, we expect that in conformal $s \ell_{n}$ Toda theory there exists a hierarchy of D-branes, which would correspond to the hierarchy of representations of the $W_{n}$ algebra which we discussed in subsection 2.2. The dimension of a D-brane would be $n-1-k$, where $k$ is the number of algebraically independent null vectors in the corresponding representation. In the case of $s \ell_{3}$ Toda theory, we would have three types of D-branes: two-dimensional continuous D-branes, onedimensional "semi-degenerate" D-branes, and zero-dimensional discrete or fully-degenerate D-branes. We will see that these expectations are fulfilled when the boundary condition is $W+\bar{W}=0$.

An important difference between the two boundary conditions $W= \pm \bar{W}$ manifests itself when analyzing the consequences of the $W_{3}$ symmetry on the correlation functions. We introduced the Ward identities which follow from the $W_{3}$ symmetry in subsection 2.3, let us now sketch how such identities constrain the correlation function of $N$ operators $V_{\alpha_{i}}\left(z_{i}\right)$ (with $\Im z_{i}>0$ ) in the presence of a boundary at $z=\bar{z}$. It turns out that the Ward identities for such an $N$-point function are identical to the Ward identities for a $2 \mathrm{~N}$ point function in the absence of a boundary, where the extra $N$ operators are "reflected" operators located at $\bar{z}_{i}$. The reflected operators are $V_{\alpha_{i}}\left(\bar{z}_{i}\right)$ if the boundary condition is $W-\bar{W}=0$, and $V_{\alpha_{i}^{*}}\left(\bar{z}_{i}\right)$ if the boundary condition is $W+\bar{W}=0$. As far as the Ward identities are concerned, we thus have the relations

$$
\begin{aligned}
& \left\langle V_{\alpha}(z)\right\rangle_{W-\bar{W}=0} \sim\left\langle V_{\alpha}(z) V_{\alpha}(\bar{z})\right\rangle, \\
& \left\langle V_{\alpha}(z)\right\rangle_{W+\bar{W}=0} \sim\left\langle V_{\alpha}(z) V_{\alpha^{*}}(\bar{z})\right\rangle .
\end{aligned}
$$

The Ward identities for a bulk two-point function $\left\langle V_{\alpha_{1}}\left(z_{1}\right) V_{\alpha_{2}}\left(z_{2}\right)\right\rangle$ are known to imply 
that it vanishes unless $\Delta_{\alpha_{1}}=\Delta_{\alpha_{2}}$ and $q_{\alpha_{1}}+q_{\alpha_{2}}=0$. Now eqs. (2.14) and (2.16) imply $\Delta_{\alpha}=\Delta_{\alpha^{*}}$ and $q_{\alpha}=-q_{\alpha^{*}}$. Therefore, while $\left\langle V_{\alpha}(z)\right\rangle_{W+\bar{W}=0}$ may be nonzero for all values of $\alpha,\left\langle V_{\alpha}(z)\right\rangle_{W-\bar{W}=0}$ must vanish unless $q_{\alpha}=0$. This restricts the momentum $\alpha$ to a one-dimensional space, which may be related to the fact that there is only one boundary parameter $\lambda_{0}$ in the case $W-\bar{W}=0$. We will however not analyze this case further, and instead concentrate on the case $W+\bar{W}=0$ from now on.

\subsection{Continuous D-branes}

Due to conformal symmetry, a one-point function on the upper half-plane must take the form

$$
\left\langle V_{\alpha}(z)\right\rangle=\frac{\mathrm{U}(\alpha)}{|z-\bar{z}|^{2 \Delta_{\alpha}}},
$$

where $\mathrm{U}(\alpha)$ is the bulk one-point structure constant, which we now want to determine. We will find constraints on $\mathrm{U}(\alpha)$ by considering the two-point function $\left\langle V_{-b \omega_{1}}(y) V_{\alpha}(z)\right\rangle$, which can be factorized in two possible ways:

$$
\begin{aligned}
\left\langle V_{-b \omega_{1}}(y) V_{\alpha}(z)\right\rangle & =\sum_{h \in H_{\omega_{1}}} C_{h}(\alpha) \mathrm{U}(\alpha-b h) \mathcal{G}_{h}(\alpha \mid y, z), \\
& =\sum_{j} R_{j} S_{j}(\alpha) \mathcal{F}_{j}(\alpha \mid y, z)
\end{aligned}
$$

Let us explain these formulas. The first formula follows from the operator product expansion

$$
V_{-b \omega_{1}} V_{\alpha} \rightarrow \sum_{h \in H_{\omega_{1}}} C_{h}(\alpha) V_{\alpha-b h}
$$

This OPE is a sum of three terms labelled by the set $H_{\omega_{1}}=\left\{\omega_{1}, \omega_{2}-\omega_{1},-\omega_{2}\right\}$ of the weights of the fundamental representation of $s \ell_{3}$; this is analogous to the tensor product of $s \ell_{3}$ representations $R_{\omega_{1}} \otimes R_{\Omega}=\sum_{h \in H_{\omega_{1}}} R_{\Omega+h}$. We choose to study the correlation function $\left\langle V_{-b \omega_{1}}(y) V_{\alpha}(z)\right\rangle$ precisely because the fully degenerate operator $V_{-b \omega_{1}}$ has such simple OPEs; we could in principle use arbitrary operators instead, but the resulting constraints on $\mathrm{U}(\alpha)$ could not necessarily be written explicitly. The OPE coefficients $C_{h}(\alpha)$ are [1]

$$
\begin{aligned}
C_{\omega_{1}}(\alpha) & =1 \\
C_{\omega_{2}-\omega_{1}}(\alpha) & =-\frac{\pi \mu}{\gamma\left(-b^{2}\right)} \frac{\gamma\left(b\left(e_{1}, \alpha-Q\right)\right)}{\gamma\left(b\left(e_{1}, \alpha\right)\right)} \\
C_{-\omega_{2}}(\alpha) & =\left(\frac{\pi \mu}{\gamma\left(-b^{2}\right)}\right)^{2} \frac{\gamma\left(b\left(e_{2}, \alpha-Q\right)\right)}{\gamma\left(b\left(e_{2}, \alpha\right)\right)} \frac{\gamma(b(\rho, \alpha-Q))}{\gamma(b(\rho, \alpha))},
\end{aligned}
$$

where we recall that $\mu$ is the bulk cosmological constant, $b$ parametrizes the central charge, and $Q=\left(b+b^{-1}\right) \rho$ (see subsection 2.2). We also introduce the function $\gamma(x)=\frac{\Gamma(x)}{\Gamma(1-x)}$ where $\Gamma(x)$ is Euler's Gamma function. The last factor in eq. (4.4) is the conformal block $\mathcal{G}_{h}(\alpha \mid y, z)$. From our remark that $N$-point functions on the upper half-plane are equivalent to $2 N$-point functions on the plane as far as Ward identities are concerned, it follows that 
$\mathcal{G}_{h}(\alpha \mid y, z)$ coincides with a bulk four-point $t$-channel conformal block,

$$
\mathcal{G}_{h}(\alpha \mid y, z)=V_{V_{-b \omega_{2}}(\bar{y})}^{V_{-b \omega_{1}}(y)} V_{V_{\alpha^{*}}(\bar{z})}
$$

Explicit expressions for such conformal blocks can be deduced from [1], where more general conformal blocks were computed. Up to simple common prefactors, the three conformal blocks are

$$
\mathcal{G}_{h}(\alpha \mid y, z) \propto\left|\frac{y-z}{y-\bar{z}}\right|_{3}^{2 b(h, \alpha-Q)}{ }_{3} F_{2}\left(\left.\begin{array}{c}
-b^{2}, \quad b\left(e_{h}, \alpha-Q\right)-b^{2}, \quad b\left(e_{h}^{\prime}, \alpha-Q\right)-b^{2} \\
b\left(e_{h}, \alpha-Q\right)+1, \quad b\left(e_{h}^{\prime}, \alpha-Q\right)+1
\end{array}|| \frac{y-z}{y-\bar{z}}\right|^{2}\right),
$$

where for a given weight $h \in H_{\omega_{1}}$ we call $e_{h}, e_{h}^{\prime}$ the two roots such that $\left(h, e_{h}\right)=\left(h, e_{h}^{\prime}\right)=1$, for instance $e_{-\omega_{2}}=-e_{2}, e_{-\omega_{2}}^{\prime}=-\rho$. Similarly, the quantity $\mathcal{F}_{j}(\alpha \mid y, z)$ in eq. (4.5) is an $s$-channel conformal block,

$$
\mathcal{F}_{j}(\alpha \mid y, z)=\sum_{V_{-b \omega_{2}}(\bar{y})}^{V_{-b \omega_{1}}(y)} \sum_{V_{\alpha^{*}}(\bar{z})}^{V_{\alpha}(z)}
$$

where $j$ labels the operator which propagates in the $s$-channel. There is a subtlety here: the $t$-channel analysis predicts the existence of three independent blocks, but only two primary operators can appear in the OPE $V_{-b \omega_{1}} V_{-b \omega_{2}}$, namely $V_{0}$ and $V_{-b \rho}$. The point is that a descendent of $V_{-b \rho}$ can appear independently of $V_{-b \rho}$ itself; we will label as $j=\rho^{\prime}$ the corresponding $s$-channel operator. In the presence of a boundary, we thus have the bulk-boundary OPE

$$
V_{-b \omega_{1}} \rightarrow \sum_{j \in\left\{0, \rho, \rho^{\prime}\right\}} R_{j} B_{-b j}
$$

where the coefficients $R_{j}$ are unknown functions of $b$, and $B_{-b j}$ are boundary operators, with the convention that $B_{-b \rho^{\prime}}$ is some descendent of $B_{-b \rho}$. We will not dwell longer on this subtlety, as we are presently only interested in the $s$-channel operator $j=0$. In this case, the bulk-boundary structure constant $S_{j}(\alpha)$ reduces to

$$
S_{0}(\alpha)=\mathrm{U}(\alpha) .
$$

We can now obtain an equation for $\mathrm{U}(\alpha)$ from the equality of (4.4) with (4.5) by using the fusion tranformation

$$
\mathcal{G}_{h}(\alpha \mid y, z)=\sum_{j} F_{h, j}(\alpha) \mathcal{F}_{j}(\alpha \mid y, z),
$$

and extracting the $\mathcal{F}_{0}$ term in eq. (4.4). The result is

$$
\sum_{h \in H_{\omega_{1}}} C_{h}(\alpha) F_{h, 0}(\alpha) \mathrm{U}(\alpha-b h)=R_{0} \mathrm{U}(\alpha) .
$$


In order to make this equation explicit, let us compute the fusing matrix elements $F_{h, 0}(\alpha)$ defined in eq. (4.15). Determining $F_{h, j}(\alpha)$ can be done by taking the limit $\Im z \rightarrow 0$ in that equation. By definition the blocks $\mathcal{F}_{j}(\alpha \mid y, z)$ are power-like functions of $\Im z$ in the limit $\Im z \rightarrow 0$, and we have

$$
\mathcal{G}_{h}(\alpha \mid y, z) \underset{\Im z \rightarrow 0}{\sim} F_{h, \rho}(\alpha)+\frac{\Im y \Im z}{|y-\bar{z}|^{2}} F_{h, \rho^{\prime}}(\alpha)+\left(\frac{\Im y \Im z}{|y-\bar{z}|^{2}}\right)^{2+3 b^{2}} F_{h, 0}(\alpha) .
$$

As the block $\mathcal{G}_{h}(\alpha \mid y, z)$ is a ${ }_{3} F_{2}$ hypergeometric function (4.11), let us study such functions, starting with their integral representation

$$
\begin{aligned}
{ }_{3} F_{2}\left(\begin{array}{ccc}
A_{1} & A_{2} & A_{3} \\
B_{1} & B_{2}
\end{array} \mid x\right)= & \frac{\Gamma\left(B_{2}\right)}{\Gamma\left(A_{3}\right) \Gamma\left(B_{2}-A_{3}\right)} \int_{0}^{1} d t t^{A_{3}-1}(1-t)^{B_{2}-A_{3}-1} \\
& \times{ }_{2} F_{1}\left(A_{1}, A_{2}, B_{1}, t x\right) .
\end{aligned}
$$

We wish to study the $x \rightarrow 1$ limit, where the critical exponents are 0,1 and $B_{1}+$ $B_{2}-A_{1}-A_{2}-A_{3}$, and to focus on the last one of those three exponents. Consider the region $t \rightarrow 1, x \rightarrow 1$, where we can use the approximation ${ }_{2} F_{1}\left(A_{1}, A_{2}, B_{1}, t x\right) \underset{t x \rightarrow 1}{\sim}$ $(1-t x)^{B_{1}-A_{1}-A_{2}} \frac{\Gamma\left(B_{1}\right) \Gamma\left(A_{1}+A_{2}-B_{1}\right)}{\Gamma\left(A_{1}\right) \Gamma\left(A_{2}\right)}$. Then we obtain the term with critical exponent $B_{1}+B_{2}-A_{1}-A_{2}-A_{3}$,

$$
\begin{aligned}
{ }_{3} F_{2}\left(\begin{array}{ccc}
A_{1} & A_{2} & A_{3} \\
B_{1} & B_{2}
\end{array} \mid z\right) \underset{z \rightarrow 1}{\supset} \frac{\Gamma\left(B_{1}\right) \Gamma\left(B_{2}\right) \Gamma\left(A_{1}+A_{2}+A_{3}-B_{1}-B_{2}\right)}{\Gamma\left(A_{1}\right) \Gamma\left(A_{2}\right) \Gamma\left(A_{3}\right)} \\
\times(1-z)^{B_{1}+B_{2}-A_{1}-A_{2}-A_{3}} .
\end{aligned}
$$

This term may or may not be the leading term of ${ }_{3} F_{2}\left(\begin{array}{ccc}A_{1} & A_{2} & A_{3} \\ B_{1} & B_{2}\end{array} \mid z\right)$ in the limit $x \rightarrow 1$, depending on the values of $A_{i}, B_{j}$. In the case of $\mathcal{G}_{h}(\alpha \mid y, z)(4.11)$, it is actually subleading, as we assume $b>0$ and therefore $B_{1}+B_{2}-A_{1}-A_{2}-A_{3}=2+3 b^{2}>2$. What we are interested in is the coefficient of $(1-z)^{2+3 b^{2}}$, which is

$$
F_{h, 0}(\alpha)=\frac{\Gamma\left(-2-3 b^{2}\right)}{\Gamma\left(-b^{2}\right)} \frac{\Gamma\left(b\left(e_{h}, \alpha-Q\right)+1\right)}{\Gamma\left(b\left(e_{h}, \alpha-Q\right)-b^{2}\right)} \frac{\Gamma\left(b\left(e_{h}^{\prime}, \alpha-Q\right)+1\right)}{\Gamma\left(b\left(e_{h}^{\prime}, \alpha-Q\right)-b^{2}\right)} .
$$

Combining this formula with the formulas for $C_{h}(\alpha)$, eq. (4.7)-(4.9), we obtain

$$
F_{h, 0}(\alpha) C_{h}(\alpha)=\frac{\Gamma\left(-2-3 b^{2}\right)}{\Gamma\left(-b^{2}\right)}\left[-\frac{\pi \mu}{\gamma\left(-b^{2}\right)}\right] \frac{A(\alpha-b h)}{A(\alpha)},
$$

where we introduced the function

$$
A(\alpha) \equiv\left[\pi \mu \gamma\left(b^{2}\right)\right]^{\frac{(\rho, \alpha-Q)}{b}} \prod_{e>0} \Gamma(b(e, \alpha-Q))^{-1} \Gamma\left(1+b^{-1}(e, \alpha-Q)\right)^{-1} .
$$

This function already appeared in conformal Toda theory [19], as a building block for the reflection coefficient $R_{w}(\alpha)=\frac{A(Q+w(\alpha-Q))}{A(\alpha)}$ defined in eq. (2.18). From that equation and 
the definition (4.3) of $\mathrm{U}(\alpha)$, it follows that $A(\alpha) \mathrm{U}(\alpha)$ must be invariant under the reflections $\alpha \rightarrow Q+w(\alpha-Q)$. The equation for $\mathrm{U}(\alpha)$ (4.16) can now be rewritten as

$$
R_{0} A(\alpha) \mathrm{U}(\alpha)=\sum_{h \in H_{\omega_{1}}} A(\alpha-b h) \mathrm{U}(\alpha-b h),
$$

where $R_{0}$ is still an unknown function of $b$, in which we actually absorbed the $\alpha$-independent prefactors of $F_{h, 0}(\alpha) C_{h}(\alpha)$ in eq. (4.21). Three more equations for $A(\alpha) \mathrm{U}(\alpha)$ can similarly be obtained, by replacing the fully degenerate operator $V_{-b \omega_{1}}$ in eqs. (4.4) and (4.5) with one of the similar operators $V_{-b \omega_{2}}, V_{-b^{-1} \omega_{1}}$ or $V_{-b^{-1} \omega_{2}}$. The resulting equations for $A(\alpha) \mathrm{U}(\alpha)$ are obtained from eq. (4.23) by replacing $H_{\omega_{1}}$ with $H_{\omega_{2}}$ and/or $b$ by $b^{-1}$. The coefficient $R_{0}$ can also change, and we rename it $\lambda_{i, \pm}$ depending on the case. So we obtain the four equations

$$
\lambda_{i, \pm} A(\alpha) \mathrm{U}(\alpha)=\sum_{h \in H_{\omega_{i}}} A\left(\alpha-b^{ \pm 1} h\right) \mathrm{U}\left(\alpha-b^{ \pm 1} h\right) .
$$

The smooth, reflection-invariant solutions of these equations are $A(\alpha) \mathrm{U}(\alpha)=$ $\sum_{w \in W} e^{(w(s), \alpha-Q)}$ where $W$ is the Weyl group and the arbitary vector $s$ is the boundary parameter. The coefficients $\lambda_{i, \pm}$ might be called the boundary cosmological constants, and their values are

$$
\lambda_{i, \pm}=\chi_{\omega_{i}}\left(-b^{ \pm 1} s\right),
$$

where we recall that the fundamental character is $\chi_{\omega_{1}}(p)=e^{\left(\omega_{1}, p\right)}+e^{\left(\omega_{2}-\omega_{1}, p\right)}+e^{-\left(\omega_{2}, p\right)}$. The full formula for the solution $U_{s}(\alpha)$ is

$$
U_{s}(\alpha)=\left[\pi \mu \gamma\left(b^{2}\right)\right]^{\frac{(\rho, Q-\alpha)}{b}} \prod_{e>0} \Gamma(b(e, \alpha-Q)) \Gamma\left(1+b^{-1}(e, \alpha-Q)\right) \sum_{w \in W} e^{(w(s), \alpha-Q)} .
$$

Our equations (4.24) being linear, this formula holds up to an $\alpha$-independent factor. We will say that $U_{s}(\alpha)$ defines a continuous D-brane when $s$ is such that $U_{s}(\alpha)$ does not diverge exponentially in the limit of large momentum $|\alpha-Q| \rightarrow \infty$. As the operators in the spectrum of $s \ell_{n}$ Toda theory have purely imaginary values of $\alpha-Q$ (see eq. (2.17)), the continuous D-branes must have real values of $s$.

Notice that we wrote the one-point structure constant $U_{s}(\alpha)$ in a form which makes sense in $s \ell_{n}$ Toda theory for arbitrary $n$, and even in conformal Toda theories based on arbitrary simply-laced Lie algebras. We conjecture that this result, and most of the results in the rest of this section, are valid in the general case and not only in $s \ell_{3}$ Toda theory.

\subsection{Degenerate D-branes}

In the previous subsection we found continuous D-branes, whose parameter space has the same dimension as the space of continuous representations. According to the classical analysis of subsection 3.4, such D-branes should be interpreted as covering the two-dimensional Toda space whose coordinates are $\phi_{1}, \phi_{2}$, because the boundary conditions for the fields $\phi_{1}, \phi_{2}$ are of the Neumann type. We will now investigate degenerate D-branes, whose dimensions and parameter spaces should be smaller. We will argue in subsection 4.3 that the dimension of a D-brane is related to the divergence of its one-point function in the 
limit $\alpha-Q \rightarrow 0$ : the higher the dimension, the more severe the divergence. So let us look for solutions $\mathrm{U}(\alpha)$ to the equations (4.24) whose divergences at $\alpha-Q \rightarrow 0$ would be less severe than the divergence of $U_{s}(\alpha)$ (4.26). Cancelling some of the divergences from the poles of the $\Gamma(b(e, \alpha-Q))$ factor can be achieved by taking a linear combination of several solutions $U_{s}(\alpha)$ with different values of $s$. However, the resulting combination will still be a solution of eq. (4.24) only provided the four parameters $\lambda_{i, \pm}$ are the same for all the involved values of $s$.

In order to find two different values $s, s^{\prime}$ which have the same parameters $\lambda_{i, \pm}$, we make two observations: first, the parameters $\lambda_{i, \pm}=\chi_{\omega_{i}}(-b s)$ (4.25) are Weyl-invariant, second, they are invariant under shifts $s \rightarrow s+2 \pi i b^{\mp 1}\left(\mathbb{Z} e_{1}+\mathbb{Z} e_{2}\right)$. Thus, there must exist two elements $w_{ \pm}$of the Weyl group such that $s-w_{ \pm}\left(s^{\prime}\right) \in 2 \pi i b^{\mp 1}\left(\mathbb{Z} e_{1}+\mathbb{Z} e_{2}\right)$. Assuming the value of $b^{2}$ to be non-rational, this restricts $s$ to a one-dimensional space. For example, the pair

$$
s=\kappa \omega_{1}+\pi i\left(\ell b+m b^{-1}\right) e_{2}, \quad s^{\prime}=\kappa \omega_{1}+\pi i\left(\ell b-m b^{-1}\right) e_{2}, \quad(\kappa, \ell, m) \in \mathbb{R} \times \mathbb{N}^{2}
$$

is such that $s-s^{\prime}=2 \pi i m b^{-1} e_{2}$ and $s-r\left(s^{\prime}\right)=2 \pi i \ell b e_{2}$. Therefore,

$$
U_{\kappa \mid \ell, m}(\alpha) \equiv U_{\kappa \omega_{1}+\pi i\left(\ell b+m b^{-1}\right) e_{2}}(\alpha)-U_{\kappa \omega_{1}+\pi i\left(\ell b-m b^{-1}\right) e_{2}}(\alpha)
$$

is a solution of eq. (4.24), and an explicit calculation yields

$$
U_{\kappa \mid \ell, m}(\alpha)=\frac{4}{A(\alpha)} \sum_{e>0} e^{\kappa\left(h_{e}, \alpha-Q\right)} \sin 2 \pi \ell b(e, \alpha-Q) \sin 2 \pi m b^{-1}(e, \alpha-Q),
$$

where to a positive root $e$ we associate the weight $h_{e} \in H_{\omega_{1}}$ such that $\left(e, h_{e}\right)=0$. In this expression the sine factors compensate some of the $\alpha-Q \rightarrow 0$ divergences of the prefactor $A(\alpha)$ (4.22). We hold that $U_{\kappa \mid \ell, m}(\alpha)$ with $\kappa \in \mathbb{R}$ and $\ell, m \in \mathbb{N}$ define the family of semi-degenerate D-branes. The boundary parameters of such D-branes are

$$
\lambda_{1,+}=e^{-\frac{2}{3} b \kappa}+2 e^{\frac{1}{3} b \kappa}(-1)^{m} \cos \pi \ell b^{2}, \quad \lambda_{1,-}=e^{-\frac{2}{3} b^{-1} \kappa}+2 e^{\frac{1}{3} b^{-1} \kappa}(-1)^{\ell} \cos \pi m b^{-2},
$$

and $\lambda_{2, \pm}$ are obtained by $\kappa \rightarrow-\kappa$.

We expect that in the more general $s \ell_{n}$ case this construction generalizes to a hierarchy of partly degenerate D-branes. The difference of two terms in eq. (4.28) should be interpreted as a sum over the $\mathbb{Z}_{2}$ subgroup of the Weyl group which leaves $\omega_{1}$ invariant, weighted by the signatures of the elements of that subgroup. In $s \ell_{n}$, there is a hierarchy of subgroups of the Weyl group which leave certain hyperplanes invariant, and summing over such subgroups should yield the partly degenerate D-branes. The case of fully degenerate D-branes corresponds to a sum over the full Weyl group, which we now study in the $s \ell_{3}$ case.

Given two integral dominant weights $\Omega, \Omega^{\prime} \in \mathbb{N} \omega_{1}+\mathbb{N} \omega_{2}$ we consider the combination

$$
U_{\Omega \mid \Omega^{\prime}}(\alpha) \equiv \sum_{w \in W} \epsilon(w) U_{2 \pi i\left(b(\Omega+\rho)+b^{-1} w\left(\Omega^{\prime}+\rho\right)\right)}(\alpha) .
$$


This can be rewritten as

$$
U_{\Omega \mid \Omega^{\prime}}(\alpha)=\frac{1}{A(\alpha)} \sum_{w \in W} \epsilon(w) e^{2 \pi i b(w(\Omega+\rho), \alpha-Q)} \sum_{w^{\prime} \in W} \epsilon\left(w^{\prime}\right) e^{2 \pi i b^{-1}\left(w^{\prime}\left(\Omega^{\prime}+\rho\right), \alpha-Q\right)} .
$$

This can be shown to have a finite limit as $\alpha-Q \rightarrow 0$, by first using the Weyl formula (2.5) in order to reduce the problem to the case of $U_{0 \mid 0}(\alpha)$,

$$
\frac{U_{\Omega \mid \Omega^{\prime}}(\alpha)}{U_{0 \mid 0}(\alpha)}=\chi_{\Omega}(2 \pi i b(\alpha-Q)) \chi_{\Omega^{\prime}}\left(2 \pi i b^{-1}(\alpha-Q)\right)
$$

and then the formula (2.6) in order to prove the regularity of $U_{0 \mid 0}(\alpha)$. We interpret $U_{\Omega \mid \Omega^{\prime}}(\alpha)$ as defining localized (zero-dimensional) D-branes, which we will call discrete D-branes or fully degenerate D-branes. But first we should check that the six values of $s$ which are involved in the sum (4.31) do have the same boundary parameters $\lambda_{i, \pm}$ (4.25). This is actually true, as a consequence of the fact that the weights $h \in H_{\omega_{1}}$ differ from one another by elements of $\mathbb{Z} e_{1}+\mathbb{Z} e_{2}$. And we find

$$
\lambda_{1,+}=e^{-2 \pi i\left(\omega_{1}, \Omega^{\prime}\right)} \chi_{\omega_{1}}\left(-2 \pi i b^{2} \Omega\right), \quad \lambda_{1,-}=e^{-2 \pi i\left(\omega_{1}, \Omega\right)} \chi_{\omega_{1}}\left(-2 \pi i b^{-2} \Omega^{\prime}\right) .
$$

In the case of discrete D-branes, these boundary cosmological constants are expected to be directly related to the values $U_{s}\left(-b^{ \pm 1} \omega_{1}\right)$ of the one-point structure constant, as was argued in the case of Liouville theory in [18]. For example, $\lambda_{1,+}$ originally appeared as the bulk-boundary structure constant $R_{0}$ in the bulk-boundary OPE of $V_{-b \omega_{1}}$ (4.13). If the one-point structure constant was normalized so that $U_{\Omega \mid \Omega^{\prime}}(0)=1$, then $\lambda_{1,+}$ would coincide with $U_{\Omega \mid \Omega^{\prime}}\left(-b^{ \pm 1} \omega_{1}\right)$. Therefore we expect

$$
\lambda_{i, \pm}=\frac{U_{\Omega \mid \Omega^{\prime}}\left(-b^{ \pm 1} \omega_{i}\right)}{U_{\Omega \mid \Omega^{\prime}}(0)} .
$$

And indeed the expressions (4.32) and (4.34) obey such relations, as can be shown with the help of the Weyl formula (2.5). (For this to be completely true we would have to reinstate the simple factor $\frac{\Gamma\left(-2-3 b^{2}\right)}{\Gamma\left(-b^{2}\right)}\left[-\frac{\pi \mu}{\gamma\left(-b^{2}\right)}\right]$ in $\lambda_{i, \pm}$; such a factor was present in eq. (4.21) but we neglected it in what followed.) It is amusing to note that we did already apply the Weyl formula to $U_{\Omega \mid \Omega^{\prime}}(\alpha)$ before, but in a different, "dual" way, in order to prove eq. (4.33).

Our expression for the bulk one-point function can be used to show that the equations of motion derived from the Lagrangian $L_{n}(2.25)$ of Toda theory are obeyed in the presence of discrete D-branes. If we identify $e^{(\alpha, \phi)}$ with the operator $V_{\alpha}$, and therefore $\phi_{i}$ with $\left.\frac{\partial}{\partial \alpha_{i}}\right|_{\alpha=0} V_{\alpha}$, then the quantum version of the equations of motion is

$$
\left.\frac{\partial}{\partial \alpha_{i}}\right|_{\alpha=0} \partial \bar{\partial}\left\langle V_{\alpha}(z)\right\rangle=\pi b \mu\left\langle V_{b e_{i}}(z)\right\rangle
$$

Using the form $\left\langle V_{\alpha}(z)\right\rangle=\frac{\mathrm{U}(\alpha)}{|z-\bar{z}|^{2 \Delta_{\alpha}}}(4.3)$ of the one-point function, this reduces to the following identity for the structure constant $\mathrm{U}(\alpha)$ :

$$
\frac{\mathrm{U}\left(b e_{i}\right)}{\mathrm{U}(0)}=\frac{2\left(b+b^{-1}\right)}{\pi b \mu} \text {. }
$$

It can be checked that this identity is obeyed by the one-point structure constant $U_{\Omega \mid \Omega^{\prime}}(\alpha)$ (4.32) of a discrete D-brane. 


\subsection{Modular bootstrap analysis}

The factorization constraint (4.4)-(4.5) whose solutions were our one-point structure constants is only one of the many equations of the conformal bootstrap formalism. Another one of these equations is relatively tractable: the modular bootstrap constraint, which relates two different decompositions of the annulus partition function

$$
\begin{aligned}
Z_{s_{1} ; s_{2}}(\tau) & =\frac{1}{6} \int d^{2} p \quad U_{s_{1}}(Q+i p) U_{s_{2}}(Q-i p) \xi_{Q+i p}(\tau), \\
& =\operatorname{Tr}_{H_{s_{1} ; s_{2}}} e^{-\frac{2 i \pi}{\tau}\left(L_{0}-\frac{c}{24}\right)} .
\end{aligned}
$$

Let us explain these formulas. We consider an annulus, the simplest Riemann surface with two boundaries. The two boundaries are characterized by their boundary parameters $s_{1}, s_{2}$, which may each correspond to any type of D-brane: continuous, semi-degenerate, or discrete; and the geometry of the annulus is characterized by the modular parameter $\tau$. The annulus partition function (or zero-point correlation function) $Z_{s_{1} ; s_{2}}(\tau)$ first has a "bulk channel" decomposition, which describes the exchange of bulk operators between the two boundaries. The resulting formula (4.38) for $Z_{s_{1} ; s_{2}}(\tau)$ therefore involves a sum over the bulk spectrum. This sum decomposes into an integral over the physical values (2.17) of the momenta $\alpha=Q+i p$ which characterize the highest-weight representations of the algebra $W_{3}$ (with the factor $\frac{1}{6}$ due to the Weyl symmetry), and sums over the descendent states in each representation, which are encoded in the characters $\xi_{\alpha}(\tau)$. The one-point structure constants $U_{s_{1}}(Q+i p)$ and $U_{s_{2}}(Q-i p)$ involve ingoing and outgoing momenta respectively, and no normalization factors appear due to the normalization assumption of [1], $\left\langle V_{Q+i p_{1}}\left(z_{1}\right) V_{Q-i p_{2}}\left(z_{2}\right)\right\rangle=\frac{\delta\left(p_{1}-p_{2}\right)}{\left|z_{12}\right|^{4 \Delta_{Q+i p_{1}}}}$ (where we write only one of the six terms of a sum over the Weyl group).

The annulus partition function also has a "boundary channel" decomposition, which describes a one-loop partition function of open strings. The resulting formula (4.39) for $Z_{s_{1} ; s_{2}}(\tau)$ is the trace over the boundary spectrum $H_{s_{1} ; s_{2}}$ of the propagator $e^{-\frac{2 i \pi}{\tau}\left(L_{0}-\frac{c}{24}\right)}$. This propagator is the operator which appears in the definition (2.19) of the characters $\xi_{\alpha}(\tau)$; in the boundary channel it appears with the dual value $-\frac{1}{\tau}$ of the modular parameter. Although we do not know the boundary spectrum $H_{s_{1} ; s_{2}}$, the modular bootstrap method will produce tests of the one-point structure constants $U_{s}(\alpha)$. This is because for some choices of $s_{1}, s_{2}$ the spectrum is discrete, and the requirement that each representation should appear with a positive integer multiplicity is a nontrivial constraint.

Let us compute $Z_{s_{1} ; s_{2}}(\tau)$ by using the bulk channel decomposition (4.38) with the bulk structure constants $U_{s}(\alpha)$ which we found in the previous two subsections. We start with the case when both D-branes are discrete, and use eq. (4.33):

$$
\begin{aligned}
Z_{\Omega_{1}\left|\Omega_{1}^{\prime} ; \Omega_{2}\right| \Omega_{2}^{\prime}}(\tau)= & \frac{1}{6} \int d^{2} p \xi_{Q+i p}(\tau) U_{0 \mid 0}(Q+i p) U_{0 \mid 0}(Q-i p) \\
& \times \chi_{\Omega_{1}^{*}}(2 \pi b p) \chi_{\Omega_{2}}(2 \pi b p) \chi_{\Omega_{1}^{\prime *}}\left(2 \pi b^{-1} p\right) \chi_{\Omega_{2}^{\prime}}\left(2 \pi b^{-1} p\right),
\end{aligned}
$$

where we used the property $\chi_{\Omega}(-p)=\chi_{\Omega^{*}}(p)$. Now let us decompose the products of characters using eq. (2.7), while computing the product $U_{0 \mid 0}(Q+i p) U_{0 \mid 0}(Q-i p)$ using 
eq. (2.6),

$$
\begin{aligned}
Z_{\Omega_{1}\left|\Omega_{1}^{\prime} ; \Omega_{2}\right| \Omega_{2}^{\prime}}(\tau)= & \frac{\left(2 \pi b^{-1}\right)^{6}}{6} \sum_{\Omega} m_{\Omega_{1}^{*}, \Omega_{2}}^{\Omega} \sum_{\Omega^{\prime}} m_{\Omega_{1}^{\prime *}, \Omega_{2}^{\prime}}^{\Omega^{\prime}} \int d^{2} p \xi_{Q+i p}(\tau) \\
& \times \chi_{\Omega}(2 \pi b p) \chi_{\Omega^{\prime}}\left(2 \pi b^{-1} p\right) \prod_{e>0} \prod_{ \pm}\left(e^{\frac{1}{2}\left(e, 2 \pi b^{ \pm 1} p\right)}-e^{-\frac{1}{2}\left(e, 2 \pi b^{ \pm 1} p\right)}\right) .
\end{aligned}
$$

The value of the (Gaussian) integral is given by eq. (2.22),

$$
Z_{\Omega_{1}\left|\Omega_{1}^{\prime} ; \Omega_{2}\right| \Omega_{2}^{\prime}}(\tau)=\frac{\left(2 \pi b^{-1}\right)^{6}}{\sqrt{3}} \sum_{\Omega, \Omega^{\prime}} m_{\Omega_{1}^{*}, \Omega_{2}}^{\Omega} m_{\Omega_{1}^{\prime *}, \Omega_{2}^{\prime}}^{\Omega^{\prime}} \xi_{-b \Omega-b^{-1} \Omega^{\prime}}\left(-\frac{1}{\tau}\right) .
$$

This is a sum of characters with positive integer coefficients $m_{\Omega_{1}^{*}, \Omega_{2}} m_{\Omega_{1}^{\prime *}, \Omega_{2}^{\prime}}^{\Omega^{\prime}}$, up to a factor which could be absorbed in a renormalization of the one-point structure constant $U_{\Omega \mid \Omega^{\prime}}(\alpha)$. The characters are those of fully degenerate representations. As we pointed out in subsection 2.2, characters $\chi_{\alpha}(\tau)$ do not fully characterize representations of the $W_{3}$ algebra, nevertheless we conjecture that the boundary spectrum is $H_{\Omega_{1}\left|\Omega_{1}^{\prime} ; \Omega_{2}\right| \Omega_{2}^{\prime}}=$ $\oplus_{\Omega, \Omega^{\prime}} m_{\Omega_{1}^{*}, \Omega_{2}}^{\Omega} m_{\Omega_{1}^{\prime *}, \Omega_{2}^{\prime}}^{\Omega^{\prime}} R_{-b \Omega-b^{-1} \Omega^{\prime}}$. If we associate the representation $R_{-b \Omega-b^{-1} \Omega^{\prime}}$ to the discrete D-brane of parameter $s=\Omega \mid \Omega^{\prime}$, fusing the representations associated to the two involved D-branes (after conjugating one of them) produces the boundary spectrum, which agrees with Cardy's ideas.

In particular, the $0 \mid 0$ D-brane corresponds to the identity representation, and for any D-brane of parameter $s$, the spectrum $H_{0 \mid 0 ; s}$ should be the single representation which is associated to that D-brane. In the case of a continuous D-brane,

$$
Z_{0 \mid 0 ; s}(\tau)=\frac{\left(2 \pi b^{-1}\right)^{6}}{6} \int d^{2} p \xi_{Q+i p}(\tau) \sum_{w \in W} e^{i(w(s), p)}=\frac{\left(2 \pi b^{-1}\right)^{6}}{\sqrt{3}} \xi_{Q-\frac{s}{2 \pi i}}\left(-\frac{1}{\tau}\right),
$$

so that the continuous representation of momentum $Q-\frac{s}{2 \pi i}$ is associated to the continuous D-brane of parameter $s$. This immediately generalizes to semi-degenerate D-branes, using the formula (4.28) for their one-point structure constants. Another generalization is to replace the identity D-brane with an arbitrary discrete D-brane,

$$
\begin{aligned}
Z_{\Omega \mid \Omega^{\prime} ; s}(\tau) & =\frac{\left(2 \pi b^{-1}\right)^{6}}{6} \int d^{2} p \xi_{Q+i p}(\tau) \chi_{\Omega^{*}}(2 \pi b p) \chi_{\Omega^{\prime *}}\left(2 \pi b^{-1} p\right) \sum_{w \in W} e^{i(w(s), p)} \\
& =\frac{\left(2 \pi b^{-1}\right)^{6}}{\sqrt{3}} \sum_{h \in H_{\Omega}} \sum_{h^{\prime} \in H_{\Omega^{\prime}}} \xi_{Q-\frac{s}{2 \pi i}}-b h-b^{-1} h^{\prime}\left(-\frac{1}{\tau}\right)
\end{aligned}
$$

which lends support to the conjecture that the boundary spectrum is obtained by fusing the representations which correspond to the two D-branes. (Notice that a weight $h$ may appear several times in $H_{\Omega}$, as happened in the definition of the character $\chi_{\Omega}(p)(2.4)$.)

\subsection{Continuous boundary spectra}

Let us investigate the boundary spectrum of $s \ell_{n}$ Toda theory in the presence of two continuous D-branes using the modular bootstrap approach. Up to numerical factors, the 
annulus partition function is formally written as

$$
Z_{s_{1} ; s_{2}}(\tau)=\int d^{n-1} p \frac{\sum_{w_{1}, w_{2} \in W} e^{i\left(w_{1}\left(s_{1}\right)-w_{2}\left(s_{2}\right), p\right)}}{\prod_{ \pm} \prod_{e>0} \sinh \pi b^{ \pm 1}(e, p)} \xi_{Q+i p}(\tau)
$$

This expression suffers from infrared divergences near $p=0$. In the case of Liouville theory $(n=2)$, the divergence is of the type $\int \frac{d p}{p^{2}}$ and therefore linear in a long distance cutoff $L$. This is attributed to the geometry of the continuous D-branes, which are supposed to extend up to infinity in the Liouville space of coordinate $\phi$. One may therefore naively expect that for general $n$ the divergence should be $L^{n-1}$. However, it is actually $L^{(n-1)^{2}}$, so there is an extra divergence $L^{(n-1)(n-2)}$ beyond what is expected on geometrical grounds.

We observe that this extra divergence is governed by the number $2 d_{n}=(n-1)(n-2)$ of parameters which are necessary to account for the infinite fusion multiplicities of continuous representations, where the factor 2 in $2 d_{n}$ is meant to take into account the antiholomorphic multiplicities. (See subsection 2.3.) Combining this observation with Cardy's ideas suggests a heuristic explanation of the divergence. Indeed, if the boundary spectrum is obtained by fusing the representations associated to the two D-branes, and fusion multplicity is infinite, then the boundary spectrum is a sum of representations with infinite multiplicities. This must then lead to divergences in the annulus partition function, in addition to the ordinary "geometrical" divergences. Therefore, we conjecture that the boundary spectrum in the presence of two continuous D-branes is the sum of all continuous representations of the $W_{n}$ algebra, each one appearing with an infinite multiplicity.

It is not obvious to us how these considerations generalize to annulus partition functions involving arbitrary D-branes. For example, in the case of $s \ell_{3}$ Toda theory with one continuous and one semi-degenerate D-brane, the annulus partition function diverges as $L^{2}$. The fusion multiplicity is finite in this case, and we conjecture that the spectrum is the sum of all continuous representations, each one appearing a finite number of times.

\section{Light asymptotic limits of some correlation functions}

The main purpose of this section is to establish a link between the classical analysis of section 3 , and the conformal bootstrap analysis of section 4 , in the case of $s \ell_{3}$ Toda theory with the boundary condition $W+\bar{W}=0$. We will use the classical solutions of the Toda equations for predicting the bulk one-point function in a certain limit, and we will find that the classical predictions agree with the bootstrap results up to unimportant details. In addition we will also predict the light asymptotic limits of the boundary two-point function in the case $W+\bar{W}=0$, and of bulk one-point and boundary two-point functions in the case $W-\bar{W}=0$, for which we did not perform the conformal bootstrap analysis. For pedagogical purposes, we will begin with the computation of the analogous correlation functions in the much simpler case of Liouville theory.

The light semi-classical asymptotic limit, or light asymptotic limit, of a correlation function $\left\langle\prod_{i} V_{\alpha_{i}}\left(z_{i}\right)\right\rangle_{s}$ in the presence of a boundary with parameter $s$, where $V_{\alpha_{i}}\left(z_{i}\right)$ may be a bulk or a boundary operator, is defined by

$$
b \rightarrow 0, \quad \eta_{i} \equiv b^{-1} \alpha_{i} \text { and } \sigma \equiv b s \text { fixed. }
$$


If the correlation function is formally represented as a functional integral over the Toda field $\phi$, with the weight $e^{-S[\phi]}$ where $S[\phi]$ is the action, then the light asymptotic limit reduces that functional integral to a finite-dimensional integral over field configurations such that $W^{(s)}=\bar{W}^{(s)}=0$ for all spins $s$ [1]. In $s \ell_{n}$ Toda theory, such field configurations are those where $X_{i}=e^{-\phi_{i}}$ are polynomials of degree $n-1$ as functions of the coordinates $z, \bar{z}$. These polynomials must obey further constraints like the boundary conditions and the reality of $\phi_{i}$. The functional integral reasoning therefore predicts that the quantity

$$
\left\langle\prod_{i} e^{\left(\eta_{i}, \phi\left(z_{i}\right)\right)}\right\rangle_{\sigma}^{\text {light }} \equiv \int_{M_{\sigma}} d \phi_{i} \prod e^{\left(\eta_{i}, \phi\left(z_{i}\right)\right)},
$$

where $M_{\sigma}$ is a finite-dimensional space of field configurations, should be related to the limit (5.1) of $\left\langle\prod_{i} V_{\alpha_{i}}\left(z_{i}\right)\right\rangle_{s}$,

$$
\lim _{b \rightarrow 0} \frac{\left\langle\prod_{i} V_{b \eta_{i}}\left(z_{i}\right)\right\rangle_{b^{-1} \sigma}}{\left\langle V_{0}(0)\right\rangle_{b^{-1} \sigma}}=\frac{\left\langle\prod_{i} e^{\left(\eta_{i}, \phi\left(z_{i}\right)\right)}\right\rangle_{\sigma}^{\text {light }}}{\langle 1\rangle_{\sigma}^{\text {light }}} .
$$

Here we normalize the correlation functions by dividing them by the partition function. This eliminates the dependences on the overall undetermined factor in the integration measure on $M_{\sigma}$, and on the value $S[\phi]$ of the action for the polynomial field configurations, which is difficult to compute. As a result, nothing in eq. (5.3) depends on the action, and we conjecture that that equation holds whether a boundary action exists or not. (See appendix B for a discussion of that point.)

\subsection{Case of Liouville theory}

Let us consider the solutions (3.4) of the Liouville equation which obey the "light asymptotic condition" $\partial^{2} X=\bar{\partial}^{2} X=0$, where $X=e^{-\phi}$. Such solutions are built from two functions $b_{1}, b_{2}$ which are polynomials of degrees at most one. Given the freedom to choose the matrix $N$, we can fix these functions without loss of generality, and we choose $b_{1}(z)=1, b_{2}(z)=z$. So we have $X(z)=(1, \bar{z}) N(\underset{z}{1})$ where $N$ is a positive Hermitian matrix of determinant one. According to equation (5.2), the one-point function of a bulk operator in the presence of a boundary with parameter $\lambda_{L}(3.6)$ is of the type

$$
\left\langle e^{\eta \phi(z)}\right\rangle_{\lambda_{L}}^{\text {light }}=\int d N \delta\left(\lambda_{L}-\frac{1}{2 i} \operatorname{Tr}(N P)\right) X(z)^{-\eta}
$$

where $d N$ is an integration measure, and we expect the boundary parameters $\lambda_{L}$ and $\sigma$ to be related.

Assuming that the integration measure $d N$ is invariant under the $S L(2, \mathbb{R})$ symmetry $N \rightarrow \Lambda^{T} N \Lambda$ (3.5), let us show that this symmetry determines the $z$-dependence of the one-point function. The subgroup of $S L(2, \mathbb{R})$ which survives our fixing of $b_{1}, b_{2}$ is the set of matrices of the type $\Lambda(x, y) \equiv\left(\begin{array}{cc}\sqrt{y} & 0 \\ -\frac{x}{\sqrt{y}} & \frac{1}{\sqrt{y}}\end{array}\right)$, which are such that $\Lambda(x, y)\left(\begin{array}{c}1 \\ x+i y\end{array}\right)=\sqrt{y}\left(\begin{array}{l}1 \\ i\end{array}\right)$. By using this residual subgroup in eq. (5.4) we obtain $\left\langle e^{\eta \phi(z)}\right\rangle_{\lambda_{L}}^{\text {light }}=(\Im z)^{-\eta}\left\langle e^{\eta \phi(i)}\right\rangle_{\lambda_{L}}^{\text {light }}$, which agrees with what we would expect from conformal symmetry. 
There is a simple method for computing the integral in eq. (5.4), which unfortunately does not easily generalize to $s \ell_{n>2}$ Toda theory. It uses the parametrization $N=\left(\begin{array}{cc}Y_{0}-Y_{1} & Y_{2}+i Y_{3} \\ Y_{2}-i Y_{3} & Y_{0}+Y_{1}\end{array}\right)$, where the constraint $\operatorname{det} N=1$ still has to be imposed. We have

$$
\left\langle e^{\eta \phi(i)}\right\rangle_{\lambda_{L}}^{\text {light }}=\int\left(\prod_{i=0}^{3} d Y_{i}\right) \delta\left(\lambda_{L}+Y_{3}\right) \delta\left(Y_{0}^{2}-Y_{1}^{2}-Y_{2}^{2}-Y_{3}^{2}-1\right)\left(2 Y_{0}-2 Y_{3}\right)^{-\eta}
$$

Integrating over $Y_{3}$ then $Y_{1}$ and $Y_{2}$, we obtain $\left\langle e^{\eta \phi(i)}\right\rangle_{\lambda_{L}}^{\text {light }}=\frac{\pi}{2^{\eta}} \int_{\sqrt{\lambda_{L}^{2}+1}}^{\infty} d Y_{0}\left(Y_{0}+\lambda_{L}\right)^{-\eta}$ from which we deduce

$$
\left\langle e^{\eta \phi(z)}\right\rangle_{\lambda_{L}}^{\text {light }}=(\Im z)^{-\eta} \frac{\pi}{2^{\eta}} \frac{\left(\lambda_{L}+\sqrt{\lambda_{L}^{2}+1}\right)^{1-\eta}}{\eta-1} .
$$

Let us now present another calculation of the integral (5.4), which can more easily be generalized to the case of $s \ell_{n}$ Toda theory with $n \geq 3$. We adopt the parametrization $N=\bar{M}^{T} M=\left(\begin{array}{cc}\rho^{-2}+|a|^{2} & \rho \bar{a} \\ \rho a & \rho^{2}\end{array}\right)$ where $M=\left(\begin{array}{cc}\rho^{-1} & 0 \\ a & \rho\end{array}\right)$ is a function of a real parameter $\rho$ and a complex parameter $a$. The integral (5.4) becomes

$$
\left\langle e^{\eta \phi(z)}\right\rangle_{\lambda_{L}}^{\text {light }}=\int \rho d \rho d^{2} a \delta\left(\lambda_{L}+\rho \Im a\right)\left(\rho^{-2}+|a+\rho z|^{2}\right)^{-\eta} .
$$

It is possible, but tedious, to compute this integral directly. Instead, let us introduce the notation

$$
\lambda_{L}=i \cosh \sigma .
$$

For the moment this is a rather awkward notation, as we have to assume that $i e^{\sigma}$ is real (and we further take it to be positive). In addition we perform the change of variables $a \rightarrow a-\rho z$, and we obtain

$$
\left\langle e^{\eta \phi(z)}\right\rangle_{\sigma}^{\text {light }}=\int \rho d \rho d^{2} a \delta\left(\frac{i}{2} e^{\sigma}+\frac{i}{2} e^{-\sigma}+\rho \Im a-\rho^{2} \Im z\right)\left(\rho^{-2}+|a|^{2}\right)^{-\eta} .
$$

In the limit

$$
\left\{\begin{array}{l}
i e^{\sigma} \rightarrow \xi i e^{\sigma} \\
z \rightarrow \xi z
\end{array}, \quad \xi \rightarrow \infty\right.
$$

the integral greatly simplifies,

$$
\left\langle e^{\eta \phi(z)}\right\rangle_{\sigma}^{\text {light }} \sim \frac{1}{\xi \Im z} \int d^{2} a\left(\frac{2 \Im z}{i e^{\sigma}}+|a|^{2}\right)^{-\eta}=(\xi \Im z)^{-\eta} \frac{\pi}{2^{\eta}} \frac{\left(i \xi e^{\sigma}\right)^{\eta-1}}{\eta-1} .
$$

This agrees with the result (5.6), in spite of the limit (5.10) which we have taken. This is because the result (5.6) has a very simple behaviour under the rescalings involved in the definition of the limit. The behaviour under the rescaling of $z$ is of course a consequence of conformal symmetry, but we have no a priori reason for the behaviour under the rescaling of $i e^{\sigma}$ to be simple. We will assume that a similar behaviour persists in $s \ell_{n}$ Toda theory with arbitrary $n$, and this will allow us to take limits analogous to (5.10) before performing integrals which would otherwise seem intractable. 
Now let us investigate whether the relation (5.3) between the classical and quantum calculations of the one-point function holds. According to eq. (5.11), the normalized classical one-point function is

$$
\frac{\left\langle e^{\eta \phi(z)}\right\rangle_{\sigma}^{\text {light }}}{\langle 1\rangle_{\sigma}^{\text {light }}}=(2 \Im z)^{-\eta} \frac{\left(i e^{\sigma}\right)^{\eta}}{1-\eta} .
$$

The behaviour of the exact Liouville one-point function (4.26) in the light asymptotic limit is

$$
\begin{aligned}
& \left\langle V_{b \eta}(z)\right\rangle_{b^{-1} \sigma} \underset{b \rightarrow 0}{\sim}(2 \Im z)^{-\eta}\left(\pi b^{-2} \mu\right)^{-\frac{\eta}{2}} \Gamma\left(\eta-b^{-2}\right) e^{|\sigma| b^{-2}} \frac{e^{|\sigma|(1-\eta)}}{\eta-1}, \\
& \frac{\left\langle V_{b \eta}(z)\right\rangle_{b^{-1} \sigma}}{\langle 1\rangle_{b^{-1} \sigma}} \underset{b \rightarrow 0}{\sim}(2 \Im z)^{-\eta}(-1)^{\eta}\left(\pi b^{2} \mu\right)^{-\frac{\eta}{2}} \frac{e^{-\eta|\sigma|}}{1-\eta}
\end{aligned}
$$

where we assume $\sigma$ to be real, and we used $\frac{\Gamma\left(\eta-b^{-2}\right)}{\Gamma\left(-b^{-2}\right)} \underset{b \rightarrow 0}{\sim}\left(-b^{-2}\right)^{\eta}$. The classical and quantum results agree, provided the cosmological constant takes the value $\mu=-\frac{1}{\pi b^{2}}(3.1)$ which was assumed in the classical analysis, and the classical boundary parameter $\sigma$ defined in eq. (5.8) is identified with the quantum boundary parameter $\sigma$ which appears in eq. (5.13). However, $i e^{\sigma}$ is assumed to be real in the classical analysis, whereas $\sigma$ is assumed to be real in the calculation of the limit of the exact one-point function. Thus, the comparison between the two methods must involve an analytic continuation of the results. This problem ultimately comes from the fact that the bulk cosmological constant $\mu$ is assumed to be negative in the classical analysis, and positive in the conformal bootstrap analysis. And the known relation [16] between the boundary cosmological constant $\lambda_{L}$ and $\sigma$ is in our notations $\left(\frac{\lambda_{L}}{\pi b^{2}}\right)^{2}=\frac{\mu}{\sin \pi b^{2}} \cosh ^{2} \sigma$, which agrees with eq. (5.8) in the $b \rightarrow 0$ limit if $\mu=-\frac{1}{\pi b^{2}}$. This confirms our earlier identification of $\lambda_{L}$ as the boundary cosmological constant, see eq. (3.8).

Finally, let us compute the light asymptotic limit of the boundary two-point function:

$$
\left\langle e^{\eta_{1} \phi\left(z_{1}\right)} e^{\eta_{2} \phi\left(z_{2}\right)}\right\rangle_{\lambda_{L}}^{\text {light }}=\int \rho d \rho d^{2} a \delta\left(\lambda_{L}+\rho \Im a\right) \prod_{i=1}^{2}\left(\rho^{-2}+\left|a+\rho x_{i}\right|^{2}\right)^{-\eta_{i}} .
$$

Let us use conformal invariance, and fix $x_{1}=0, x_{2}=\infty$. This makes the computation elementary, and the result is

$$
\left\langle e^{\eta_{1} \phi(0)} e^{\eta_{2} \phi(\infty)}\right\rangle_{\lambda_{L}}^{\text {light }}=\delta\left(\eta_{1}-\eta_{2}\right) \frac{\Gamma\left(\eta_{1}-\frac{1}{2}\right)}{\Gamma\left(\eta_{1}\right)}\left(\lambda_{L}^{2}+1\right)^{\frac{1}{2}-\eta_{1}}
$$

This agrees with the expectations from the conformal bootstrap analysis [16], provided our relation (5.8) between the classical and quantum boundary parameters is assumed.

\subsection{Case of $s \ell_{3}$ Toda theory with $W-\bar{W}=0$}

Let us consider the solutions (3.13) of the $s \ell_{3}$ Toda equations which obey the "light asymptotic condition" $\partial^{3} X_{i}=\bar{\partial}^{3} X_{i}=0$. Such solutions are built from three functions $b_{1}, b_{2}, b_{3}$ which are polynomials of degrees at most two. Given the freedom to 
choose the matrix $N$, we can fix these functions without loss of generality, and we choose $B(z) \equiv\left(b_{1}(z), b_{2}(z), b_{3}(z)\right)=\left(\frac{1}{2} z^{2}, z, 1\right)$. The corresponding Wronskians are $\left(w_{1}(z), w_{2}(z), w_{3}(z)\right)=\left(1,-z, \frac{1}{2} z^{2}\right)$ and they obey $w_{i}=P_{i j} b_{j}$ with $P \equiv\left(\begin{array}{ccc}0 & 0 & 1 \\ 0 & -1 & 0 \\ 1 & 0 & 0\end{array}\right)$. This leads to

$$
X_{1}=B(\bar{z}) N B(z)^{T}, \quad X_{2}=B(\bar{z}) P N^{-1 T} P B(z)^{T} .
$$

According to equation (5.2), the one-point function of a bulk operator $e^{(\eta, \phi(z))}=$ $e^{\eta_{1} \phi_{1}(z)+\eta_{2} \phi_{2}(z)}$ in the presence of a boundary with parameter $\lambda_{0}(3.15)$ is of the type

$$
\left\langle e^{(\eta, \phi(z))}\right\rangle_{\lambda_{0}}^{\text {light }}=\int d N \delta\left(\lambda_{0}-\operatorname{det} \frac{1}{2}\left(N+N^{T}\right)\right) X_{1}^{-\eta_{1}} X_{2}^{-\eta_{2}}
$$

We may use the $\operatorname{SL}(3, \mathbb{R})$ symmetry $N \rightarrow \Lambda N \Lambda^{T}$ (3.14) in order to determine the dependence of the one-point function on $z=x+i y$. Introducing the family of matrices $\Lambda(x, y) \equiv\left(\begin{array}{ccc}y^{-1} & -x y^{-1} & \frac{1}{2} x^{2} y^{-1} \\ 0 & 1 & -x \\ 0 & 0 & y\end{array}\right)$, we have $\Lambda(x, y)^{T} P \Lambda(x, y)=P$ and $\Lambda(x, y) B(x+i y)^{T}=$ $y B(i)^{T}$. Together with the assumption that the measure $d N$ in eq. (5.18) is invariant under the symmetry, this implies $\left\langle e^{(\eta, \phi(z))}\right\rangle_{\lambda_{0}}^{\text {light }}=(\Im z)^{-2 \eta_{1}-2 \eta_{2}}\left\langle e^{(\eta, \phi(i))}\right\rangle_{\lambda_{0}}^{\text {light }}$. The power of $\Im z$ thus obtained is $-2(\rho, \eta)=\lim _{b \rightarrow 0}\left(-2 \Delta_{b \eta}\right)$ where $\Delta_{\alpha}$ given by eq. (2.14), as expected from conformal symmetry. Now it turns out that the $\operatorname{SL}(3, \mathbb{R})$ symmetry can yield further information on the one-point function. This is because after assuming $z=i$ there still is a residual subgroup of matrices $\Lambda(d, e, f) \equiv\left(\begin{array}{ccc}f & -2 d & 2 f-2 e \\ d & e & -2 d \\ \frac{1}{2} f-\frac{1}{2} e & d & f\end{array}\right)$ where $d, e, f$ are real parameters constrained by $\operatorname{det} \Lambda(d, e, f)=\left(e^{2}+4 d^{2}\right)(2 f-e)=1$, and such matrices obey the relations $\Lambda(d, e, f)^{-1 T} B(i)^{T}=\frac{1}{e-2 i d} B(i)^{T}$ and $\Lambda(d, e, f) P B(i)^{T}=(e+2 i d) P B(i)^{T}$. Thus, under transformations $N \rightarrow \Lambda^{-1} N \Lambda^{-1 T}$, we have $X_{1}(i) \rightarrow \frac{1}{e^{2}+4 d^{2}} X_{1}(i)$ and $X_{2}(i) \rightarrow\left(e^{2}+4 d^{2}\right) X_{2}(i)$. This shows that $\left\langle e^{(\eta, \phi(i))}\right\rangle_{\lambda_{0}}^{\text {light }}$ must vanish unless $\eta_{1}=\eta_{2}$. The consequences of the $\mathrm{SL}(3, \mathbb{R})$ symmetry may be summarized as

$$
\left\langle e^{(\eta, \phi(z))}\right\rangle_{\lambda_{0}}^{\text {light }}=\delta\left(\eta_{1}-\eta_{2}\right)(\Im z)^{-4 \eta_{1}}\left\langle e^{\eta_{1}(\rho, \phi(z))}\right\rangle_{\lambda_{0}}^{\text {light }} .
$$

Let us now introduce a parametrization $N=\bar{M}^{T} M$ in terms of a triangular matrix $M=\left(\begin{array}{lll}\rho & a & b \\ 0 & \nu & c \\ 0 & 0 & \tau\end{array}\right)$ which depends on three real parameters $\rho, \nu, \tau$ such that $\rho \nu \tau=1$, and three complex parameters $a, b, c$. Then $N=\left(\begin{array}{ccc}\rho^{2} & \rho a & \rho b \\ \rho \bar{a} & \nu^{2}+|a|^{2} & \bar{a} b+\nu c \\ \rho \bar{b} & a \bar{b}+\nu \bar{c} & |b|^{2}+|c|^{2}+\tau^{2}\end{array}\right)$ and

$$
\begin{aligned}
& d N=\frac{1}{\pi^{3}} \rho^{3} d \rho \nu d \nu d^{2} a d^{2} b d^{2} c, \\
& X_{1}=\tau^{2}+|c+\nu z|^{2}+\left|b+a z+\frac{1}{2} \rho z^{2}\right|^{2}, \\
& X_{2}=\rho^{-2}+\left|\tau a+\nu^{-1} z\right|^{2}+\left|a c-\nu b+\rho c z+\frac{1}{2} \tau^{-1} z^{2}\right|^{2} .
\end{aligned}
$$

In terms of such variables, the boundary parameter is

$$
\lambda_{0}=\operatorname{det} \frac{1}{2}\left(N+N^{T}\right)=\rho^{2}\left[(\nu \Im b-\Re c \Im a)^{2}+\left(\nu^{2}+\Im a^{2}\right)\left(\tau^{2}+\Im c^{2}\right)\right] .
$$


We consider the one-point function (5.18) and perform the simultaneous shifts

$$
a \rightarrow a-\rho z, \quad b \rightarrow b-a z+\frac{1}{2} \rho z^{2}, \quad c \rightarrow c-\nu z,
$$

thereby obtaining

$$
\begin{aligned}
\left\langle e^{(\eta, \phi(z))}\right\rangle_{\lambda_{0}}^{\text {light }}=\int \frac{d N}{\rho^{2}}\left(\tau^{2}+|b|^{2}+|c|^{2}\right)^{-\eta_{1}}\left(\rho^{-2}+\tau^{2}|a|^{2}+|a c-\nu b|^{2}\right)^{-\eta_{2}} \\
\quad \delta\left(\frac{\lambda_{0}}{\rho^{2}}-[\nu \Im b-\Re c \Im a+\Im z(\rho \Re c-\nu \Re a)]^{2}-\left[\nu^{2}+(\Im a-\rho \Im z)^{2}\right]\left[\tau^{2}+(\Im c-\nu \Im z)^{2}\right]\right) .
\end{aligned}
$$

In the limit

$$
\left\{\begin{array}{l}
\lambda_{0} \rightarrow \xi^{4} \lambda_{0} \\
z \rightarrow \xi z
\end{array}, \quad \xi \rightarrow \infty\right.
$$

the integral simplifies and reduces to

$$
\begin{aligned}
\left\langle e^{(\eta, \phi(z))}\right\rangle_{\lambda_{0}}^{\text {light }} & \sim \frac{1}{\xi^{4}}(\Im z)^{-2 \eta_{1}-2 \eta_{2}} \lambda_{0}^{\frac{\eta_{1}+\eta_{2}-2}{2}} \int \frac{d \nu}{\nu} \nu^{\eta_{1}-\eta_{2}} \times I_{\eta_{1}, \eta_{2}}, \\
I_{\eta_{1}, \eta_{2}} & \equiv \frac{1}{\pi^{3}} \int d^{2} a d^{2} b d^{2} c\left(1+|b|^{2}+|c|^{2}\right)^{-\eta_{1}}\left(1+|a|^{2}+|a c-b|^{2}\right)^{-\eta_{2}} .
\end{aligned}
$$

The integral $I_{\eta_{1}, \eta_{2}}$ can be computed for arbitrary values of $\eta_{1}, \eta_{2}$ by making repeated use of the formula $\frac{1}{\pi} \int d^{2} z\left(|z|^{2}+A\right)^{-\eta}=\frac{A^{1-\eta}}{\eta-1}$, with the result

$$
I_{\eta_{1}, \eta_{2}}=\frac{1}{\left(\eta_{1}-1\right)\left(\eta_{2}-1\right)\left(\eta_{1}+\eta_{2}-2\right)}=\prod_{e>0} \frac{1}{(e, \eta-\rho)} .
$$

Using this result in the particular case $\eta_{1}=\eta_{2}$, we obtain the expression for the one-point function in the limit (5.26),

$$
\xi^{4}\left\langle e^{(\eta, \phi(z))}\right\rangle_{\lambda_{0}}^{\text {light }} \sim \delta\left(\eta_{1}-\eta_{2}\right)(\Im z)^{-4 \eta_{1}} \frac{\lambda_{0}^{\eta_{1}-1}}{\left(\eta_{1}-1\right)^{3}} .
$$

By analogy with the case of Liouville theory, we conjecture that this is the light asymptotic limit of the one-point function for all values of $\lambda_{0}$ and $z$ (up to a possible redefinition of $\left.\lambda_{0}\right)$, and not just in the limit (5.26). What we have rigorously established is however only the presence of the $\delta\left(\eta_{1}-\eta_{2}\right)(\Im z)^{-4 \eta_{1}}$ factor, which follows from the $\operatorname{SL}(3, \mathbb{R})$ symmetry. This is already significant evidence that our classical and conformal bootstrap analyses of the $W-\bar{W}=0$ boundary condition actually describe the same D-branes.

We conclude with a few words on the boundary two-point function $\left\langle e^{(\eta, \phi(x))} e^{(\mu, \phi(y))}\right\rangle_{\lambda_{0}}^{\text {light }}$. The $\mathrm{SL}(3, \mathbb{R})$ symmetry determines its dependence on the boundary coordinates $x, y$, and implies that it must vanish unless the momenta of the two boundary operators are conjugate to each other, $\eta=\mu^{*}$. This relation between the two momenta can be confirmed by a direct calculation using the parametrization (5.20)-(5.22), which yields

$$
\begin{aligned}
& \left\langle e^{(\eta, \phi(0))} e^{(\mu, \phi(\infty))}\right\rangle_{\lambda_{0}}^{\text {light }}=\delta^{(2)}\left(\eta-\mu^{*}\right) \int d^{2} a d^{2} b d^{2} c \\
& \delta\left(\lambda_{0}-\left(\Im a^{2}+1\right)\left(\Im c^{2}+1\right)-(\Im b-\Re c \Im a)^{2}\right)\left(1+|b|^{2}+|c|^{2}\right)^{-\eta_{1}}\left(1+|a|^{2}+|a c-b|^{2}\right)^{-\eta_{2}} .
\end{aligned}
$$


This integral is the same as $I_{\eta_{1}, \eta_{2}}$ (5.28) with an additional delta-function, and at this moment we do not know how to compute it. Also, we have no conformal bootstrap results to compare it with. In the special case $\lambda_{0}=-1$ when the boundary action is local (3.17), the exact result is known [19], but does not have a light asymptotic limit. Correspondingly, our light asymptotic calculation is meaningful only if $\lambda_{0} \geq 1$, because if $\lambda_{0}<1$ the argument of the $\delta$ function in eq. (5.31) cannot vanish.

\subsection{Case of $s \ell_{3}$ Toda theory with $W+\bar{W}=0$}

Let us consider the solutions (3.19) of the $s \ell_{3}$ Toda equations which obey the "light asymptotic condition" $\partial^{3} X_{i}=\bar{\partial}^{3} X_{i}=0$. We will write them in terms of the same functions $B(z)=\left(\frac{1}{2} z^{2}, z, 1\right)$ as in the $W-\bar{W}=0$ case, together with the same matrix $P=\left(\begin{array}{ccc}0 & 0 & 1 \\ 0 & -1 & 0 \\ 1 & 0 & 0\end{array}\right)$ which relates them to their Wronskians,

$$
X_{1}=B(\bar{z}) N P B(z)^{T}, \quad X_{2}=B(\bar{z}) P N^{-1 T} B(z)^{T} .
$$

Now that we choose these particular functions $B(z)$, it is possible to ensure that $\phi_{i}=$ $-\log X_{i}$ are real by imposing the simple condition that $N P$ be a positive Hermitian matrix. This restricts the $\operatorname{SL}(3, \mathbb{C})$ symmetry group $(3.20)$ to its $\operatorname{SL}(3, \mathbb{R})$ subgroup, which still acts as $N \rightarrow \Lambda^{T} N \Lambda^{-1 T}$. (Beware that the matrix $P$ transforms nontrivially under this symmetry.) In addition, this implies that the eigenvalues of $N$ must be real, because there is a matrix $M$ such that $N P=\bar{M}^{T} M$, and $N$ is thus conjugate to the Hermitian matrix $M P \bar{M}^{T}$.

According to eq. (5.2), the one-point function of a bulk operator $e^{(\eta, \phi(z))}=$ $e^{\eta_{1} \phi_{1}(z)+\eta_{2} \phi_{2}(z)}$ in the presence of a boundary with parameters $\lambda_{1}, \lambda_{2}(3.21)$ is of the type

$$
\left\langle e^{(\eta, \phi(z))}\right\rangle_{\lambda_{1}, \lambda_{2}}^{\text {light }}=\int d N \delta\left(\lambda_{1}-\operatorname{Tr} N\right) \delta\left(\lambda_{2}-\operatorname{Tr} N^{-1}\right) X_{1}^{-\eta_{1}} X_{2}^{-\eta_{2}}
$$

The consequences of the $\operatorname{SL}(3, \mathbb{R})$ symmetry on this integral can be evaluated using the same particular symmetry transformations as in the case $W-\bar{W}=0$. Using $\Lambda(x, y)$ we similarly obtain the expected dependence on $z$. Using $\Lambda(d, e, f)$ however teaches us nothing new, because such transformations now leave $X_{1}, X_{2}$ invariant.

Let us now use the parametrization $N=\bar{M}^{T} M P$ in terms of an upper-triangular matrix $M=\left(\begin{array}{lll}\rho & a & b \\ 0 & \nu & c \\ 0 & 0 & \tau\end{array}\right)$ which depends on three real parameters $\rho, \nu, \tau$ such that $\rho \nu \tau=1$, and three complex parameters $a, b, c$. The expressions (5.20)-(5.22) for $d N, X_{1}, X_{2}$ still hold, and we find

$$
\begin{aligned}
& \lambda_{1}=\operatorname{Tr} N=2 \rho \Re b-\nu^{2}-|a|^{2}, \\
& \lambda_{2}=\operatorname{Tr} N^{-1}=2 \rho \nu \Re(a c)-2 \rho \nu^{2} \Re b-\rho^{2}|c|^{2}-\rho^{2} \tau^{2} .
\end{aligned}
$$

We perform the shifts (5.24) in the expression (5.33) for the one-point function, and obtain

$$
\begin{aligned}
\left\langle e^{(\eta, \phi(z))}\right\rangle_{\lambda_{1}, \lambda_{2}}^{\text {light }}= & \int d N\left(\tau^{2}+|b|^{2}+|c|^{2}\right)^{-\eta_{1}}\left(\rho^{-2}+\tau^{2}|a|^{2}+|\nu b-c a|^{2}\right)^{-\eta_{2}} \\
& \times \delta\left(\lambda_{1}+\nu^{2}+|a|^{2}-2 \rho \Re b+2 \rho^{2}(\Im z)^{2}-4 \Im a \Im z\right) \\
& \times \delta\left(\lambda_{2}+\nu^{2} \lambda_{1}+\nu^{4}+\nu^{-2}+|\rho c-\nu \bar{a}-2 i \rho \nu \Im z|^{2}\right)
\end{aligned}
$$


We do not know how to perform this integral, except by taking a particular limit of the variables $z, \lambda_{1}, \lambda_{2}$. To this end, we parametrize the eigenvalues of $N$ as $\left\{e^{-(h, \sigma)}\right\}=$ $\left\{e^{-\sigma_{1}}, e^{\sigma_{1}-\sigma_{2}}, e^{\sigma_{2}}\right\}$ for a vector $\sigma=\sigma_{1} e_{1}+\sigma_{2} e_{2}$ in the Cartan subalgebra of $s \ell_{3}$. Then the parameters $\lambda_{i}$ coincide with values of the fundamental and antifundamental characters of $s \ell_{3}$,

$$
\lambda_{1}=\chi_{\omega_{2}}(\sigma), \quad \lambda_{2}=\chi_{\omega_{1}}(\sigma) .
$$

Notice however that the matrix $N$ is not positive, and $\sigma_{1}, \sigma_{2}$ are not expected to be real numbers. Rather, we assume that $e^{\sigma_{1}}$ and $e^{\sigma_{2}}$ are negative real numbers. We now introduce the limit

$$
\left\{\begin{array}{l}
z \rightarrow \xi z \\
e^{\sigma_{i}} \rightarrow \xi^{2} e^{\sigma_{i}}
\end{array}, \quad \xi \rightarrow \infty,\right.
$$

so that $\lambda_{i} \rightarrow \xi^{2} e^{\sigma_{i}}$. In this limit, the delta-functions in the integral (5.36) fix the variables $\rho, \nu, \tau$ to values proportional to the eigenvalues of $N$, namely

$$
\rho^{2}=-\frac{1}{2} \frac{1}{(\Im z)^{2}} e^{\sigma_{2}}, \quad \nu^{2}=\frac{1}{2} e^{\sigma_{1}-\sigma_{2}}, \quad \tau^{2}=-4(\Im z)^{2} e^{-\sigma_{1}} .
$$

And we find that the integral takes the value

$$
\begin{aligned}
& \left\langle e^{(\eta, \phi(z))}\right\rangle_{\sigma}^{\text {light }} \sim \frac{1}{\xi^{4}}(\Im z)^{-2 \eta_{1}-2 \eta_{2}}\left(-e^{\sigma_{1}}\right)^{\eta_{1}-1}\left(-e^{\sigma_{2}}\right)^{\eta_{2}-1} I_{\eta_{1}, \eta_{2}}, \\
& \frac{\left\langle e^{(\eta, \phi(z))}\right\rangle_{\sigma}^{\text {light }}}{\langle 1\rangle_{\sigma}^{\text {light }}}=\frac{-2}{(\Im z)^{2(\rho, \eta)}}\left(-e^{\sigma_{1}}\right)^{\eta_{1}}\left(-e^{\sigma_{2}}\right)^{\eta_{2}} \prod_{e>0} \frac{1}{(e, \eta-\rho)},
\end{aligned}
$$

where we used the definition (5.28) and value (5.29) of the integral $I_{\eta_{1}, \eta_{2}}$. We derived this result in the case of $s \ell_{3}$ Toda theory, but it is not very difficult to generalize it to the case of $s \ell_{n}$ Toda theory with arbitrary $n$. By analogy with Liouville theory, we conjecture that this result holds for general values of $z$ and $\sigma$, and not just in the limit (5.38).

Let us investigate the light asymptotic limit (5.1) of the conformal bootstrap result (4.26). The behaviour of the factor $\sum_{w \in W} e^{(w(s), \alpha-Q)}$ depends on which Weyl chamber $s=b^{-1} \sigma$ belongs to; there is a Weyl chamber such that

$$
\begin{aligned}
& \left\langle V_{b \eta}(z)\right\rangle_{b^{-1} \sigma} \underset{b \rightarrow 0}{\sim} \frac{\left(\pi \mu b^{-2}\right)^{-(\rho, \eta)}}{(2 \Im z)^{2(\rho, \eta)}} e^{-b^{-2}(\rho, \sigma)} e^{(\eta-\rho, \sigma)} \prod_{e>0} \frac{\Gamma\left(1+(e, \eta-\rho)-b^{-2}(e, \rho)\right)}{(e, \eta-\rho)}, \\
& \frac{\left\langle V_{b \eta}(z)\right\rangle_{b^{-1} \sigma}}{\left\langle V_{0}(i)\right\rangle_{b^{-1} \sigma}} \underset{b \rightarrow 0}{\sim}-2 \frac{\left(\pi \mu b^{2}\right)^{-(\rho, \eta)}}{(\Im z)^{2(\rho, \eta)}}(-1)^{2(\rho, \eta)} e^{(\eta, \sigma)} \prod_{e>0} \frac{1}{(e, \eta-\rho)},
\end{aligned}
$$

which generalizes the Liouville result (5.13). Checking the agreement (5.3) between the classical calculation (5.41) and the conformal bootstrap result (5.43) involves assuming that the boundary parameters $e^{\sigma_{i}}$ which we introduced in both analyses coincide. Then the boundary cosmological constants $\lambda_{i,+}(4.25)$ agree with the classical boundary parameters $\lambda_{1,2}$ (5.37). However, as in Liouville theory, we must analytically continue the onepoint function, because the boundary parameters $e^{\sigma_{i}}$ take positive values in the bootstrap analysis and negative values in the classical calculation. 
This agreement between the classical and bootstrap analyses, and the identification of their respective boundary parameters, have interesting consequences in the case when $\sigma$ belongs to the boundary of a Weyl chamber, that is $(e, \sigma)=0$ for $e$ some positive root. This is the case when two of the eigenvalues $\left\{e^{-\sigma_{1}}, e^{\sigma_{1}-\sigma_{2}}, e^{\sigma_{2}}\right\}$ of the matrix $N$ coincide; in the classical analysis of subsection 3.4 this corresponded to the D-brane being one-dimensional. In the conformal bootstrap analysis, this case corresponds to the semidegenerate D-branes, as is clear from eq. (4.30) for the boundary cosmological constants, where two of the three terms coincide. In the limit $b \rightarrow 0$ with $c=-\frac{1}{3} b \kappa$ fixed and $m$ odd, the boundary cosmological constants become $\left\{\begin{array}{l}\lambda_{1,+}=e^{2 c}-2 e^{-c} \\ \lambda_{2,+}=e^{-2 c}-2 e^{c}\end{array}\right.$. This allows us to identify $c$ with the position of the D-brane, as given by the Dirichlet conditions eq. (3.22) from the classical analysis.

We conclude with a few words on the boundary two-point function $\left\langle e^{(\eta, \phi(x))} e^{(\mu, \phi(y))}\right\rangle_{\lambda_{1}, \lambda_{2}}^{\text {light }}$. The $\mathrm{SL}(3, \mathbb{R})$ symmetry determines its dependence on the boundary coordinates $x, y$, and implies that it must vanish unless the momenta obey $(\rho, \eta-\mu)=0$. This condition can be interpreted as the conformal invariance of the boundary theory; however, assuming the momenta to be related to the $W^{(3)}$ charges as in eq. (2.16), we would expect a stronger constraint from the full $W_{3}$ symmetry, namely $\eta=\mu^{*}$. And indeed this is the constraint we found in the $W-\bar{W}=0$ case. But we can confirm the absence of this constraint in the present case by the explicit calculation

$$
\begin{aligned}
& \left\langle e^{(\eta, \phi(0))} e^{(\mu, \phi(\infty))}\right\rangle_{\lambda_{1}, \lambda_{2}}^{\text {light }}=\delta((\rho, \eta-\mu)) \int d^{2} a d^{2} b d^{2} c \frac{d \nu}{\nu} \nu^{2\left(\eta_{1}-\mu_{2}\right)} \\
& \times \delta\left(\lambda_{1}-2 \nu^{-1} \Re b+\nu^{2}+\nu^{2}|a|^{2}\right) \delta\left(\lambda_{2}+2 \nu \Re b+\nu^{-2}+\nu^{-2}|c|^{2}-2 \nu \Re(a c)\right) \\
& \times\left(1+|b|^{2}+|c|^{2}\right)^{-\eta_{1}}\left(1+|a|^{2}+|a c-b|^{2}\right)^{-\eta_{2}} .
\end{aligned}
$$

Curiously, the limit in which we are able to compute this integral is different from the limit (5.38) which we used in the case of the one-point function, and in particular no longer forbids the coincidence of two eigenvalues of $N$. This limit is chosen so that a rescaling of $\nu$ can match the behaviours of $\lambda_{1}$ and $\lambda_{2}$ in the delta-functions:

$$
\left\{\begin{array}{l}
e^{\sigma_{1}} \rightarrow \xi^{-1} e^{\sigma_{1}} \\
e^{\sigma_{2}} \rightarrow \xi e^{\sigma_{2}}
\end{array}, \quad \xi \rightarrow \infty\right.
$$

We obtain in this limit

$$
\begin{aligned}
\xi^{3+2 \eta_{2}-2 \mu_{1}}\left\langle e^{(\eta, \phi(0))} e^{(\mu, \phi(\infty))}\right\rangle_{\sigma}^{\text {light }} \sim & \delta((\rho, \eta-\mu)) \frac{1}{\left(\eta_{2}-1\right)\left(\mu_{2}-1\right)} \frac{\Gamma\left(\eta_{1}+\eta_{2}-\frac{3}{2}\right)}{\Gamma\left(\eta_{1}+\eta_{2}-1\right)} \\
& \times\left|e^{\sigma_{2}-\sigma_{1}}\right|^{\mu_{1}-\eta_{2}-\frac{3}{2}}\left|\sinh \frac{1}{2}\left(\sigma_{1}+\sigma_{2}\right)\right|^{-2 \eta_{1}-2 \eta_{2}+3}
\end{aligned}
$$

This result is invariant under the exchange of the two operators $\eta \leftrightarrow \mu$. On the other hand there is no invariance under $\eta \rightarrow \eta^{*}, \mu \rightarrow \mu^{*}$. This is because our limit (5.45) treats the boundary parameters $\lambda_{1}, \lambda_{2}$ in an asymmetric way; in particular $\lambda_{1}$ and $\lambda_{2}$ do not go to infinity at the same rate. 


\begin{tabular}{|l|l|c|c|c|c|}
\hline Theory & Type of Brane & $d$ & Parameters & Classical parameters & Action \\
\hline \multirow{2}{*}{ Liouville } & Continuous & 1 & $s \in \mathbb{R}$ & $\lambda_{L}=i \cosh b s$ & $\lambda_{L} \int e^{\phi}$ \\
& Discrete & 0 & $\ell \mid \ell^{\prime} \in \mathbb{N}^{2}$ & n. a. & n. a. \\
\hline \multirow{2}{*}{$s \ell_{3}, W=\bar{W}$} & Continuous & 2 & $?$ & $\lambda_{0}$ & nonlocal \\
& Degenerate & $?$ & $?$ & $?$ & $?$ \\
\hline \multirow{3}{*}{$s \ell_{3}, W=-\bar{W}$} & Continuous & 2 & $s \in \mathbb{R}^{2}$ & $\lambda_{i}=\chi_{\omega_{i}}(b s)$ & inexistent \\
& Semi-degenerate & 1 & $\kappa \mid \ell, m \in \mathbb{R} \times \mathbb{N}^{2}$ & $c=-\frac{1}{3} b \kappa$ & 0 \\
& Discrete & 0 & $\ell, m \mid \ell^{\prime}, m^{\prime} \in \mathbb{N}^{4}$ & n. a. & n. a. \\
\hline
\end{tabular}

Table 1. Summary of results.

\section{Conclusion}

Combining classical and conformal bootstrap analyses yields a consistent picture of the moduli space of maximally symmetric D-branes in $s \ell_{n}$ conformal Toda theory. Our results and conjectures on these moduli spaces and on the existence of a boundary action in the $s \ell_{2}$ and $s \ell_{3}$ cases are summarized in table 1 .

In the case of the boundary conditions $W^{(s)}-\bar{W}^{(s)}=0$, the dimension of the moduli space is the integer part of $\frac{n}{2}$ (subsection 3.3). This coincides with the number of nonzero charges $\left\{q^{(s)}\right\}_{2 \leq s \leq n-1 \mid s}$ even for bulk operators whose one-point functions do not vanish (section 4), in accordance with a generalization of Cardy's idea. In the $s \ell_{3}$ case, we provide predictions for certain correlation functions in the light asymptotic limit, namely the bulk one-point function (5.30) and boundary two-point function (5.31).

We examined the case of the boundary conditions $W^{(s)}-(-1)^{s} \bar{W}^{(s)}=0$ in more detail. We propose that there exists a hierarchy of D-branes of dimensions $d=0 \cdots n-1$, which correspond to the partly degenerate representations of the $W_{n}$ algebra. In particular, there are continuous D-branes of dimension $n-1$, and discrete D-branes of dimension 0 . The moduli space of $d$-dimensional D-branes is itself $d$-dimensional, although there are also discrete parameters. This was the result of classical (subsection 3.4) and bootstrap (section 4) analyses, which were shown to agree in detail (subsection 5.3). In particular, we found explicit formulas for the bulk one-point functions of continuous (4.26) and discrete (4.32) D-branes. In the $s \ell_{3}$ case, we also computed the bulk one-point functions of the semi-degenerate D-branes (4.29). As our D-branes conform to Cardy's ideas by corresponding to representations of the $W_{n}$ algebra, they also correspond to the topological defects of the very interesting article [20] (where such defects are related to certain operators in four-dimensional gauge theories). And a D-brane's one-point function is closely related to the corresponding defect operator's coefficients.

The calculation of annulus partition functions leads to natural conjectures for the spectra of open strings with one end on a discrete D-brane (subsection 4.3). These spectra coincide with what can be obtained by fusing the two representations which correspond to the two involved D-branes. If this structural property persists in the case of all D-branes, then it can help explain the divergences of the annulus partition functions (subsection 4.4). Infinite fusion multiplicities indeed appear in the fusion of two continuous representations 
(subsection 2.3), so that we expect infinite multiplicities in the spectra of continuous Dbranes. This might also explain the apparent violation of the $W_{3}$ symmetry in the minisuperspace prediction (5.46) for the boundary two-point function. A boundary spectrum with infinite multiplicities can certainly not be adequately parametrized by momenta $\alpha=b \eta$, and an operator with a given momentum might correspond to a combination of states belonging to different representations of the $W_{3}$ algebra.

Thus, the moduli space of D-branes may now be well-understood, but the boundary operators and their correlation functions remain problematic, and they certainly have new and complicated features.

\section{Acknowledgments}

We are grateful to Alexei Litvinov and Philippe Roche for interesting discussions. Moreover, we wish to thank Philippe Roche and Volker Schomerus for comments on the draft of this article.

\section{A Minisuperspace limits of some correlation functions}

In addition to the light asymptotic limit which we studied in section 5, there is another semi-classical limit in which Toda correlation functions simplify and can in certain cases be independently predicted: the minisuperspace limit, where our two-dimensional field theory reduces to a one-dimensional system. In this limit, a bulk primary operator $V_{Q+i p}(z)$ corresponds to a wavefunction $\Psi_{p}(\phi)$, which is a solution of the Schrödinger equation of Toda quantum mechanics [1],

$$
\left[-\left(\frac{\partial}{\partial \phi}\right)^{2}+2 \pi \mu b^{-2} \sum_{i=1}^{n-1} e^{\left(e_{i}, \phi\right)}\right] \Psi_{p}(\phi)=p^{2} \Psi_{p}(\phi) .
$$

(Compare with the $s \ell_{n}$ Toda Lagrangian $L_{n}(2.25)$, and notice the rescaling $\phi \rightarrow b^{-1} \phi$.) Here the variable $\phi$ can be interpreted as the $z$-independent zero-mode of the Toda field $\phi(z)$. The Schrödinger equation is deduced from the Hamiltonian picture of the dynamics of $\phi$, which is associated to radial quantization in the $z$-plane.

In the minisuperspace limit, a boundary with parameter $\sigma$ corresponds to a boundary wavefunction $\Psi_{\sigma}^{\mathrm{bdy}}(\phi)$, which can be interpreted as the density of the corresponding Dbrane. If a boundary Lagrangian $L^{\mathrm{bdy}}[\phi]$ is known, then the boundary wavefunction can be obtained by computing this Lagrangian for constant values of the field $\phi(z)$, namely

$$
\Psi_{\sigma}^{\mathrm{bdy}}(\phi)=e^{-L^{\mathrm{bdy}}(\phi)} .
$$

In any case, the minisuperspace one-point function is defined as

$$
\left\langle\Psi_{p}\right\rangle_{\sigma}^{\mathrm{mini}} \equiv \int d \phi \Psi_{p}(\phi) \Psi_{\sigma}^{\mathrm{bdy}}(\phi),
$$

and we expect that it is related to a $b \rightarrow 0$ limit of the one-point function $\left\langle V_{\alpha}(z)\right\rangle_{s}$,

$$
\lim _{b \rightarrow 0}(\Im z)^{2 \Delta_{Q+i b p}}\left\langle V_{Q+i b p}(z)\right\rangle_{b^{-1} \sigma}=\left\langle\Psi_{p}\right\rangle_{\sigma}^{\operatorname{mini}}
$$


(Compare with the light asymptotic limit (5.3).)

In the case of Liouville theory, a boundary Lagrangian is known. Then it is possible to compute the minisuperspace one-point function (A.3) and to compare it with the conformal bootstrap one-point function. It turns out that eq. (A.4) is obeyed, which provides a test of the conformal bootstrap one-point function [16]. In the case of $s \ell_{3}$ Toda theory with $W+\bar{W}=0$, no boundary action exists, as we will see in appendix B. We will reason in the opposite direction, and deduce the minisuperspace boundary wavefunction $\Psi_{\sigma}^{\text {bdy }}(\phi)$ from the conformal bootstrap one-point function. We will do this first in Liouville theory, as a preparation for the case of $s \ell_{3}$ Toda theory. The boundary wavefunction will turn out to have interesting properties; in particular it provides the generating function of the Bäcklund transformation which maps the Toda classical mechanics of $\phi$ to the free classical mechanics of $\sigma$. As we saw in section 3, there is a good reason why the Toda boundary parameter $\sigma$ can be interpreted as a free field: there exists a Bäcklund transformation from conformal Toda theory to a free field theory, such that the $W+\bar{W}=0$ boundary conditions in Toda theory are mapped to Dirichlet boundary conditions in the free theory, and the Toda boundary parameter $\sigma$ is mapped to the free field boundary parameter, which is the boundary value of the free field.

\section{A.1 Case of Liouville theory}

In this case the Schrödinger equation (A.1) becomes $\left[-\frac{1}{2} \frac{\partial^{2}}{\partial \phi^{2}}+2 \pi \mu b^{-2} e^{2 \phi}\right] \Psi_{p}(\phi)=$ $\frac{1}{2} p^{2} \Psi_{p}(\phi)$. The solution is [16]

$$
\Psi_{p}(\phi)=\left(\pi \mu b^{-2}\right)^{-\frac{i}{2} p} \frac{2}{\Gamma(-i p)} K_{i p}\left(2 \sqrt{\pi \mu b^{-2}} e^{\phi}\right),
$$

where $K_{\nu}(z)$ is a Bessel function, and $\Psi_{p}$ is normalized so that

$$
\int d \phi \Psi_{p_{1}}(\phi) \Psi_{p_{2}}(\phi)=2 \pi \delta\left(p_{1}+p_{2}\right) .
$$

The minisuperspace limit (A.4) of the Liouville one-point function (4.26) is

$$
\left\langle\Psi_{p}\right\rangle_{\sigma}^{\operatorname{mini}}=2\left(\pi \mu b^{-2}\right)^{-\frac{i}{2} p} \Gamma(i p) \cos (p \sigma) .
$$

According to eq. (A.2) and eq. (A.3) we can deduce the boundary wavefunction from the knowledge of $\left\langle\Psi_{p}\right\rangle_{\sigma}^{\mathrm{mini}}$,

$$
\Psi_{\sigma}^{\mathrm{bdy}}(\phi)=\frac{1}{2 \pi} \int d p\left\langle\Psi_{-p}\right\rangle_{\sigma}^{\mathrm{mini}} \Psi_{p}(\phi)=\frac{2}{\pi} \int d p \cos (p \sigma) K_{i p}\left(2 \sqrt{\pi \mu b^{-2}} e^{\phi}\right) .
$$

The calculation is performed using the formula $\int_{0}^{\infty} d p \cos (a p) K_{i p}(z)=\frac{\pi}{2} e^{-z \cosh a}$, with the result

$$
\Psi_{\sigma}^{\mathrm{bdy}}(\phi)=e^{-L_{2}^{\mathrm{bdy}}}, \quad L_{2}^{\mathrm{bdy}}=\sqrt{4 \pi \mu b^{-2}} \cosh (\sigma) e^{\phi} .
$$

The function $L_{2}^{\mathrm{bdy}}(\phi, \sigma)$ generates a canonical transformation between Toda and free classical mechanics, as follows from the identity

$$
\left(\frac{\partial L_{2}^{\mathrm{bdy}}}{\partial \phi}\right)^{2}-\left(\frac{\partial L_{2}^{\mathrm{bdy}}}{\partial \sigma}\right)^{2}=4 \pi \mu b^{-2} e^{2 \phi}
$$


whose right hand-side is the bulk Liouville potential, see the Lagrangian (2.25). Considering indeed $\phi, \sigma$ as time-dependent variables with associated momenta $\dot{\phi}=-\frac{\partial L_{2}^{\text {bdy }}}{\partial \phi}$ and $\dot{\sigma}=$ $\frac{\partial L_{2}^{\text {bdy }}}{\partial \sigma}$, the Liouville equation of motion $\ddot{\phi}=4 \pi \mu b^{-2} e^{2 \phi}$ amounts to the $\phi$-derivative of eq. (A.10), and the free equation of motion $\ddot{\sigma}=0$ amounts to the $\sigma$-derivative of eq. (A.10).

\section{A.2 Case of $s \ell_{3}$ Toda theory with $W+\bar{W}=0$}

The solution of the Schrödinger equation (A.1) in the case of $s \ell_{3}$ Toda theory is $[1,21]$

$$
\begin{aligned}
& \Psi_{p}(\phi)=\frac{8\left(\pi \mu b^{-2}\right)^{-i(\rho, p)}}{\prod_{e>0} \Gamma(-i(e, p))} e^{\frac{i}{6}\left(e_{1}-e_{2}, \phi\right)\left(e_{1}-e_{2}, p\right)} \\
& \times \int_{0}^{\infty} \frac{d t}{t} t^{i\left(e_{2}-e_{1}, p\right)} K_{i(\rho, p)}\left(2 \sqrt{1+t^{-2}} \sqrt{\frac{\pi \mu}{b^{2}}} e^{\frac{1}{2}\left(e_{1}, x\right)}\right) K_{i(\rho, p)}\left(2 \sqrt{1+t^{2}} \sqrt{\frac{\pi \mu}{b^{2}}} e^{\frac{1}{2}\left(e_{2}, x\right)}\right),
\end{aligned}
$$

and it is normalized such that

$$
\int d^{2} \phi \Psi_{p_{1}}(\phi) \Psi_{p_{2}}(\phi)=(2 \pi)^{2} \delta^{(2)}\left(p_{1}+p_{2}\right) .
$$

The minisuperspace limit (A.4) of the $s \ell_{3}$ Toda one-point function (4.26) is

$$
\left\langle\Psi_{p}\right\rangle_{\sigma}^{\operatorname{mini}}=\left(\pi \mu b^{-2}\right)^{-i(\rho, p)} \prod_{e>0} \Gamma(i(e, p)) \sum_{w \in W} e^{i(w(\sigma), p)} .
$$

According to eq. (A.2) and eq. (A.3) we can deduce the boundary wavefunction from the knowledge of $\left\langle\Psi_{p}\right\rangle_{\sigma}^{\operatorname{mini}}$,

$$
\Psi_{\sigma}^{\mathrm{bdy}}(\phi)=\frac{1}{(2 \pi)^{2}} \int d^{2} p\left\langle\Psi_{-p}\right\rangle_{\sigma}^{\operatorname{mini}} \Psi_{p}(\phi)
$$

The calculation can be performed using the formula

$$
\int_{0}^{\infty} d p \cos (p \sigma) K_{i p}\left(z_{1}\right) K_{i p}\left(z_{2}\right)=\frac{\pi}{2} K_{0}\left(\sqrt{z_{1}^{2}+z_{2}^{2}+2 b c \cosh \sigma}\right) .
$$

The result is

$$
\Psi_{\sigma}^{\mathrm{bdy}}(\phi)=K_{0}\left(L_{3}^{(0)}\right)
$$

where we define

$$
\begin{aligned}
L_{3}^{(0)} & \equiv \sqrt{4 \pi \mu b^{-2}} \sqrt{e^{\left(e_{1}, \phi\right)}+e^{\left(e_{2}, \phi\right)}+e^{\left(\omega_{1}, \phi\right)} \chi_{\omega_{1}}\left(\sigma^{*}\right)+e^{\left(\omega_{2}, \phi\right)} \chi_{\omega_{2}}\left(\sigma^{*}\right)} \\
& =\sqrt{4 \pi \mu b^{-2}} \prod_{h \in H_{\omega_{1}}} \sqrt{e^{\frac{1}{3}\left(e_{1}, \phi\right)}+(-1)^{(\rho, h)} e^{\frac{1}{3}\left(e_{2}, \phi\right)} e^{\left(h, \sigma^{*}\right)}} .
\end{aligned}
$$

In the strong coupling region where $\mu$ is large, we have $\Psi_{\sigma}^{\text {bdy }}(\phi) \sim e^{-L_{3}^{(0)}}$ as follows from $K_{0}(z) \underset{z \rightarrow \infty}{\sim} \sqrt{\frac{\pi}{2 z}} e^{-z}$. And $L_{3}^{(0)}$ generates the canonical transformation from Toda classical 
mechanics to the free classical mechanics. (The transformation itself is written in [22].) As in the case of Liouville theory, this follows from the identity

$$
\left(\frac{\partial L_{3}^{(0)}}{\partial \phi}\right)^{2}-\left(\frac{\partial L_{3}^{(0)}}{\partial \sigma}\right)^{2}=4 \pi \mu b^{-2}\left[e^{\left(e_{1}, \phi\right)}+e^{\left(e_{2}, \phi\right)}\right]
$$

which can be proved with the help of the formulas

$$
\left\{\begin{array}{l}
\left(\frac{\partial \chi_{\omega_{1}}(\sigma)}{\partial \sigma}\right)^{2}=\frac{2}{3} \chi_{2 \omega_{1}}(\sigma)-\frac{4}{3} \chi_{\omega_{2}}(\sigma) \\
\left(\frac{\partial \chi_{\omega_{1}}(\sigma)}{\partial \sigma}, \frac{\partial \chi_{\omega_{2}}(\sigma)}{\partial \sigma}\right)=\frac{1}{3} \chi_{\rho}(\sigma)-\frac{8}{3}
\end{array}, \quad\left\{\begin{array}{l}
\chi_{\omega_{1}}^{2}=\chi_{2 \omega_{1}}+\chi_{\omega_{2}} \\
\chi_{\omega_{1}} \chi_{\omega_{2}}=\chi_{\rho}+1
\end{array}\right.\right.
$$

To conclude, let us come back to the interpretation of the boundary wavefunction $\Psi_{\sigma}^{\text {bdy }}(\phi)$ as the density of the continuous D-brane of parameter $\sigma$, as suggested by eq. (A.3). In the weak coupling region where $L_{3}^{(0)}$ is small, we have $\Psi_{\sigma}^{\text {bdy }}(\phi) \underset{(\rho, \phi) \rightarrow-\infty}{\sim}-(\rho, \phi)$ as follows from $K_{0}(z) \underset{z \rightarrow 0}{\sim}-\log \frac{z}{2}$. Thus, the density of the D-brane grows linearly with $\phi$. The minisuperspace annulus partition function $Z_{\sigma_{1} ; \sigma_{2}}^{\operatorname{mini}}=\int d^{2} \phi \Psi_{\sigma_{1}}(\phi) \Psi_{\sigma_{2}}(\phi)$ therefore has an $L^{4}$ infrared divergence, where $L$ is a large distance cutoff. This confirms the divergence which was found by modular bootstrap methods in subsection 4.3. This contrasts with the case of Liouville theory, where the density of a continuous D-brane is constant in the weak coupling region, and correspondingly the annulus partition function diverges as $L$, which is the volume of the $\phi$-space in that case.

\section{B On the existence of a boundary action in $s \ell_{3}$ Toda theory}

The functional integral formalism is often useful in the study of conformal field theories, although in general it permits the calculation of only a subset of the correlation functions. In this formalism, correlation functions are expressed as functional integrals over the fields $\phi_{i}$, where field configurations come with weights $e^{-S}$. Here $S$ is the action, which may or may not be written as the integral of a certain Lagrangian $L$, namely $S=\int d^{2} z L(z)$. If the Lagrangian exists and is local, that is if $L(z)$ is a function of the fields $\phi_{i}$ and finitely many of their derivatives at the point $z$, then the action is also called local. If the space has a boundary $z=\bar{z}$, the boundary action or boundary terms of the action are the terms which depend only on the values of the fields at the boundary, and local boundary actions are those of the type $S=\int_{z=\bar{z}} d x L(x)$ where $L(x)$ is a local boundary Lagrangian.

The choice of an action $S$ is constrained by the classical theory. Namely, the solutions of the classical equations of motion and boundary conditions should be functional critical points of the action. This constraint does not fully determine $S$; here we will however only be concerned with the question of the existence of at least one action which obeys this constraint.

In $s \ell_{3}$ conformal Toda theory on surfaces with no boundaries, the Lagrangian $L_{3}(2.25)$ is known [1]. In Liouville theory on surfaces with boundaries, we have the boundary Lagrangian $L_{2}^{\text {bdy }}(3.8)$, see [16]. In the case of $s \ell_{3}$ Toda theory, we could so far derive our 
boundary conditions from boundary Lagrangians only in particular subcases of the two cases $W= \pm \bar{W}$. We will now investigate systematically for which boundary conditions $(W= \pm \bar{W})$ and boundary parameters $\left(\lambda_{0}\right.$ or $\left.\lambda_{1}, \lambda_{2}\right)$ boundary actions can exist.

\section{B.1 Boundary conditions as functional one-forms}

Let us assume the existence of a boundary action $S^{\text {bdy }}\left[\phi_{i}\right]$, that is a functional of the values of the Toda fields $\phi_{1}, \phi_{2}$ at the boundary. We however do not assume that $S^{\text {bdy }}\left[\phi_{i}\right]$ is local. In particular we do not forbid introducing auxiliary boundary fields in addition to $\phi_{i}$, so long as these auxiliary fields can be eliminated using their equations of motion. We only exclude the possibility for fields to obey Dirichlet boundary conditions, which excludes the particular case (3.22) from the analysis.

Let us derive the Neumann-type boundary conditions from the action $S=$ $\int d^{2} z \frac{1}{2}(\partial \phi, \bar{\partial} \phi)+S_{3}^{\mathrm{bdy}}\left[\phi_{i}\right]$, where the interaction terms in the bulk action (2.25) can be omitted as they will not contribute. We find

$$
\frac{1}{2 i}(\partial-\bar{\partial})\left(2 \phi_{1}-\phi_{2}\right)=\frac{\delta S^{\text {bdy }}}{\delta \phi_{1}}, \quad \frac{1}{2 i}(\partial-\bar{\partial})\left(2 \phi_{2}-\phi_{1}\right)=\frac{\delta S^{\text {bdy }}}{\delta \phi_{2}} .
$$

In terms of the $X_{i}=e^{-\phi_{i}}$, this becomes

$$
2 \frac{(\partial-\bar{\partial}) X_{1}}{X_{1}^{2}}-\frac{(\partial-\bar{\partial}) X_{2}}{X_{1} X_{2}}=2 i \frac{\delta S^{\mathrm{bdy}}}{\delta X_{1}}, \quad 2 \frac{(\partial-\bar{\partial}) X_{2}}{X_{2}^{2}}-\frac{(\partial-\bar{\partial}) X_{1}}{X_{1} X_{2}}=2 i \frac{\delta S^{\mathrm{bdy}}}{\delta X_{2}} .
$$

The existence of the boundary action $S^{\text {bdy }}$ can now be interpreted as the condition that the functional one-form

$$
g=\left(2 \frac{(\partial-\bar{\partial}) X_{1}}{X_{1}^{2}}-\frac{(\partial-\bar{\partial}) X_{2}}{X_{1} X_{2}}\right) \delta X_{1}+\left(2 \frac{(\partial-\bar{\partial}) X_{2}}{X_{2}^{2}}-\frac{(\partial-\bar{\partial}) X_{1}}{X_{1} X_{2}}\right) \delta X_{2}
$$

be exact, namely $g=\delta\left(2 i S^{\text {bdy }}\right)$. It follows that $g$ must be closed, $\delta g=0$. In order to be able to work with this condition, we will study functional calculus in the next subsection.

Before that, let us point out that the natural variables to work with are not $X_{1}, X_{2}$ but the functions $b_{1}, b_{2}, b_{3}$ in terms of which we wrote the solutions of the Toda equations (3.13) and (3.19). These variables are subject to the constraint $\mathrm{Wr}\left[b_{1}, b_{2}, b_{3}\right]=1$, so that we must include the possibility of such constraints in our study of functional calculus.

\section{B.2 Technical interlude: functional calculus}

We wish to study functional forms which depend on functions $b_{i}(x)$. A zero-form is a functional $S\left[b_{i}\right]$. A one-form is an object $g=\int d x \sum_{i} g_{i}(x) \delta b_{i}(x)$, where $g_{i}(x)$ are $x$ dependent functionals of $b_{i}$. An example of a one-form is the differential of a zero-form, namely $\delta S=\int d x \sum_{i} \frac{\delta S}{\delta b_{i}(x)} \delta b_{i}(x)$. A two-form is an object $k=\int d x d y \sum_{i j} k_{i j}(x, y) \delta b_{i}(x) \wedge$ $\delta b_{j}(y)$, where $k_{i j}(x, y)$ are $x, y$-dependent functionals of $b_{i}$. The basic two-forms $\delta b_{i}(x) \wedge$ $\delta b_{j}(y)=-\delta b_{j}(y) \wedge \delta b_{i}(x)$ are antisymmetric, which however does not imply the vanishing 
of $\delta b_{i}(x) \wedge \delta b_{i}(y)=-\delta b_{i}(y) \wedge \delta b_{i}(x)$. So the differential of a one-form is

$$
\begin{aligned}
\delta\left(\sum_{i} \int d x g_{i}(x) \delta b_{i}(x)\right)= & \sum_{i<j} \int d x d y\left(\frac{\delta g_{i}(x)}{\delta b_{j}(y)}-\frac{\delta g_{j}(y)}{\delta b_{i}(x)}\right) \delta b_{i}(x) \wedge \delta b_{j}(y) \\
& +\sum_{i} \int d x d y \frac{\delta g_{i}(x)}{\delta b_{i}(y)} \delta b_{i}(x) \wedge \delta b_{i}(y) .
\end{aligned}
$$

As an exercise, we can compute the differential of an action functional $S=$ $\int d x L\left(b(x), b^{\prime}(x)\right)$,

$$
\delta S=\int d z d y\left[\frac{\partial L}{\partial b}(z) \delta(z-y)+\frac{\partial L}{\partial b^{\prime}}(z) \delta^{\prime}(z-y)\right] \delta b(y)=\int d z\left[\frac{\partial L}{\partial b}-\frac{\partial}{\partial z} \frac{\partial L}{\partial b^{\prime}}\right](z) \delta b(z),
$$

and we can check that $\delta^{2} S=0$.

Now we will be interested in variables $b_{1}, b_{2}, b_{3}$ which are not independent, as they obey the constraint $\mathrm{Wr}\left[b_{1}, b_{2}, b_{3}\right]=1$. If these were ordinary variables instead of functions, the condition for the form $g=\sum g_{i} d b_{i}$ to be closed modulo a constraint $C\left(b_{1}, b_{2}, b_{3}\right)=1$ would simply be $d g \wedge d C=0$, and the integral $S$ of the one-form $g$ would be characterized by $(d S-g) \wedge d C=0$. Let us generalize these notions to the case of functional forms. Let $g=\int d x \sum_{i=1}^{3} g_{i}(x) \delta b_{i}(x)$ be a one-form, let us study the condition that it is closed modulo the constraint Wr. We denote $\delta g=\int d x d y \sum_{i j} k_{i j}(x, y) \delta b_{i}(x) \wedge \delta b_{j}(y)$ with $k_{i j}(x, y)=$ $-k_{j i}(y, x)$.

We assume for a moment that the constraint can be inverted and rewritten as $b_{3}=$ $\phi\left[b_{1}, b_{2}\right]$. Then it is straightforward to rewrite $g=\int d x \sum_{i=1}^{2} \tilde{g}_{i}(x) \delta b_{i}(x)$ and to compute $\delta g$ in terms of $\phi$ and $k_{i j}$. We find that the vanishing of $\delta g$ modulo the constraint $\operatorname{Wr}\left[b_{1}, b_{2}, b_{3}\right]=$ 1 is equivalent to

$$
\begin{aligned}
K_{12}-K_{13}-K_{32}+K_{33}=K_{11}-K_{13}-K_{31}+K_{33} & =K_{22}-K_{23}-K_{32}+K_{33}=0 \\
\text { where } K_{i j} & \equiv\left(\frac{\delta \mathrm{Wr}}{\delta b_{i}}\right)^{-1 t} k_{i j}\left(\frac{\delta \mathrm{Wr}}{\delta b_{j}}\right)^{-1}
\end{aligned}
$$

In the definition of $K_{i j}$ we have used new notations for functions of two variables $f(x, y)$ such as $\frac{\delta \mathrm{Wr}(x)}{\delta b_{i}(y)}$ or $k_{i j}(x, y)$. Namely, the products and inverses of such functions are defined with respect to the product law $\left(f_{1} f_{2}\right)(x, y) \equiv \int d z f_{1}(x, z) f_{2}(z, y)$, and the transposition is defined as the exchange of the two variables, $f^{t}(x, y) \equiv f(y, x)$. In the case when the functions $b_{i}$ are $x$-independent, the product law becomes commutative, the objects $k_{i i}$ and $K_{i i}$ vanish, and the conditions (B.6) boil down to $K_{12}+K_{23}+K_{31}=0$ which is equivalent to $d g \wedge d \mathrm{Wr}=0$ as we found by the direct analysis of that case. Notice that the conditions (B.6) on the matrix $K_{i j}$ are equivalent to $\sum_{i j} v_{i} K_{i j} v_{j}^{\prime}=0$ for any two vectors $v, v^{\prime}$ such that $\sum_{i} v_{i}=\sum_{i} v_{j}^{\prime}=0$.

Then the conditions for an "action" functional $S$ to be the integral of the functional one-form $g$ modulo the constraint $\mathrm{Wr}$ is:

$$
\left(\frac{\delta S}{\delta b_{1}}-g_{1}\right)\left(\frac{\delta \mathrm{Wr}}{\delta b_{1}}\right)^{-1}=\left(\frac{\delta S}{\delta b_{2}}-g_{2}\right)\left(\frac{\delta \mathrm{Wr}}{\delta b_{2}}\right)^{-1}=\left(\frac{\delta S}{\delta b_{3}}-g_{3}\right)\left(\frac{\delta \mathrm{Wr}}{\delta b_{3}}\right)^{-1} .
$$


Now the Wronskian constraint is not invertible, as $b_{3}$ cannot be fully determined in terms of $b_{1}, b_{2}$. So the quantities $\left(\frac{\delta \mathrm{Wr}_{i}}{\delta b_{i}}\right)^{-1}$ are ambiguous. We indeed find that $\frac{\delta \mathrm{Wr}_{\mathrm{r}}}{\delta b_{i}}$ has several inverses, parametrized by numbers $\kappa_{j k}^{i}$,

$$
\left(\frac{\delta \mathrm{Wr}}{\delta b_{i}}\right)^{-1}(x, y)=\frac{1}{w_{i}^{2}}\left[\Theta(y-x) \epsilon_{i j k} b_{j}(x) b_{k}(y)+\sum_{j, k \neq i} \kappa_{j k}^{i} b_{j}(x) b_{k}(y)\right],
$$

where $\Theta(x)$ is a step function such that $\Theta^{\prime}(x)=\delta(x)$, and we recall the notations $w_{i}=\epsilon_{i j k} b_{j} b_{k}^{\prime}$ and $\mathrm{Wr}=\epsilon_{i j k} b_{i} b_{j}^{\prime} b_{k}^{\prime \prime}$. Then for $\delta g$ to vanish modulo the constraint, the condition (B.6) must hold for all values of $\kappa_{j k}^{i}$. Similarly, integrating the functional oneform $g$ modulo the constraint requires the equation (B.8) to be satisfied for all values of $\kappa_{j k}^{i}$.

\section{B.3 Existence of the boundary action if $W-\bar{W}=0$}

We have found that the boundary conditions $W-\bar{W}=0$ lead to the expressions (3.13) for the Toda fields $X_{1}, X_{2}$ in terms of functions $b_{i}$ subject to the Wronskian constraint. The expressions (3.16) for $(\partial-\bar{\partial}) X_{i}$ are also known. These expressions depend on a constant matrix $N_{i j}$ of size 3 and determinant 1; it will be convenient to decompose both $N$ and $N^{-1 T}$ into symmetric and antisymmetric parts, according to $N_{i j}=S_{i j}+\epsilon_{i j k} A_{k}$ and $N^{-1 T}=\sigma_{i j}+\epsilon_{i j k} \alpha_{k}$.

So we can compute the one-form $g$ (B.3) and its differential $k=\delta g$ in terms of the functions $b_{i}$. Taking the ambiguities $\kappa_{j k}^{i}$ to vanish in the inversion (B.9) of the Wronskian constraint, the quantities $K_{i j}$ (B.7) turn out to be of the form

$$
K_{i j}(x, y)=\frac{2 \epsilon_{i \ell m} \epsilon_{j p q} b_{\ell}(x) b_{p}(y)}{w_{i}^{2}(x) w_{j}^{2}(y)} \int d z \Theta(x-z) \Theta(y-z) \Lambda_{m q}^{i j}(z),
$$

where we sum over repeated indices except $i, j$, and the tensor $\Lambda_{m q}^{i j}$, which is defined for $q \neq j$ and $m \neq i$ and obeys $\Lambda_{m q}^{i j}=-\Lambda_{q m}^{j i}$, is

$$
\begin{aligned}
\Lambda_{m q}^{i j}= & \frac{2 \sigma_{q r} w_{r} w_{j} b_{m}}{X_{1} X_{2}^{2}}\left(\alpha_{i} S_{u v}-\alpha_{u} S_{i v}\right) b_{u} b_{v}-\frac{2 \sigma_{m r} w_{r} w_{i} b_{q}}{X_{1} X_{2}^{2}}\left(\alpha_{j} S_{u v}-\alpha_{u} S_{j v}\right) b_{u} b_{v} \\
& +\frac{2 S_{i r} b_{r} w_{j} b_{m}}{X_{1}^{2} X_{2}}\left(A_{u} \sigma_{q v}-A_{q} \sigma_{u v}\right) w_{u} w_{v}-\frac{2 S_{j r} b_{r} w_{i} b_{q}}{X_{1}^{2} X_{2}}\left(A_{u} \sigma_{m v}-A_{m} \sigma_{u v}\right) w_{u} w_{v} \\
& +\left(\alpha_{i} S_{j r}-\alpha_{j} S_{i r}\right) \frac{b_{r} b_{m} b_{q}}{X_{1} X_{2}}+\left(A_{m} \sigma_{q r}-A_{q} \sigma_{m r}\right) \frac{w_{i} w_{j} w_{r}}{X_{1} X_{2}}
\end{aligned}
$$

In the special case of the free boundary conditions we have $A_{m}=\alpha_{m}=0$ thus $\Lambda_{m q}^{i j}=0$. In the special case when the boundary Lagrangian given by eq. (3.17) we have $S_{i j}=U_{i} U_{j}$ thus $\alpha_{i} S_{u v}-\alpha_{u} S_{i v}=A_{u} \sigma_{q v}-A_{q} \sigma_{u v}=0$ thus again $\Lambda_{m q}^{i j}=0$.

For a quantity $K_{i j}$ of the form (B.10), the condition (B.6) amounts to

$$
\left\{\begin{array} { l } 
{ \forall m \neq i } \\
{ \forall q \neq j }
\end{array} , \int \Lambda _ { m q } ^ { i j } = 0 \text { and } \left\{\begin{array}{l}
\forall j, k \\
\forall i \neq m
\end{array}, \epsilon_{j p q} \frac{b_{p}(y)}{w_{j}^{2}(y)} \int^{y} \Lambda_{m q}^{i j}=\epsilon_{k p q} \frac{b_{p}(y)}{w_{k}^{2}(y)} \int^{y} \Lambda_{m q}^{i k}\right.\right.
$$


where $\int^{y} \Lambda$ is the primitive of the function $\Lambda$. Curiously, taking into account the ambiguities parametrized by $\kappa_{j k}^{i}$ does not yield extra equations.

We wish to find out whether the equation (B.12) holds for any triples $\left(b_{1}, b_{2}, b_{3}\right)$ obeying the Wronskian constraint. We do not know how to do this except by testing the equation for a number of triples. Large families of solutions of the Wronskian constraint can be built from functions of the type $z^{\nu}$ or $e^{\nu z}$. This raises the questions of the admissible behaviour of $b_{i}(z)$ at $z=\infty$ and at generic points $z$, and of the appropriate contours of integration in our equation (B.12). We have no satisfactory answers to these questions. So we will test only the purely algebraic consequences of our equation.

Consider an equation of the type $u_{0}(y)=0$ where $u_{0}(y)=\sum_{i=1}^{n} u_{i}(y) \int^{y} v_{i}$. Let us build the matrix of size $n+1$ formed by $u_{i}$ and their first $n$ derivatives, $M=\left[u_{i}^{(j)}\right]_{i, j=0 \cdots n}$. Then $\operatorname{det} M=0$ is a purely algebraic consequence of the original equation, in the sense that the terms involving primitives $\int^{y} v_{i}$ cancel. Applying this treatment to eq. (B.12) removes the need to deal with integrals and to worry about the regularity of $b_{i}(z)$. For all the numerous cases which we tested, we found that the condition $\operatorname{det} M=0$ held. This is strong evidence that the form $\delta g$ is closed. This is strong evidence that it is in fact exact, and we conjecture that there exists a boundary action from which the boundary condition (3.12) can be derived.

This action is expected to be a functional $S^{\text {bdy }}$ of the values of the Toda fields $\phi_{1}, \phi_{2}$ at the boundary $z=\bar{z}$. In addition, $S^{\text {bdy }}$ is expected to depend on the boundary parameter $\lambda_{0}$. Comparing its definition (B.2) with the formulas (3.13) for $X_{i}$ and (3.16) for $(\partial-\bar{\partial}) X_{i}$, we see that $S^{\text {bdy }}$ cannot be local, that is of the type $\int L^{\text {bdy }}\left[\phi_{1}, \phi_{2}\right]$ where $L^{\text {bdy }}$ is a function of $\phi_{i}$ and finitely many of their derivatives, except in the two special cases $\lambda_{0}= \pm 1$ which we considered in subsection 3.3. It is possible that the nonlocal boundary action has a simple expression as a local functional of $b_{i}$. Even so, it would not be very easy to use such an action in free-field computations of correlation functions.

\section{B.4 No boundary action if $W+\bar{W}=0$}

We have found that the boundary condition $W+\bar{W}=0$ led to the expressions (3.19) for the Toda fields $X_{1}, X_{2}$ in terms of functions $b_{i}$ subject to the Wronskian constraint. Expressions for $(\partial-\bar{\partial}) X_{i}$ can easily be derived. These expressions depend on a constant matrix $N_{i j}$ of size 3 and determinant 1 ; it is not restrictive to assume that $N$ is diagonal with eigenvalues $\nu_{1}, \nu_{2}, \nu_{3}$.

The rest of the reasoning is similar to the case $W-\bar{W}=0$, with a different formula for the object $\Lambda_{m q}^{i j}$ which appears in eq. (B.10):

$$
\begin{aligned}
\Lambda_{m q}^{i j}= & \left(\nu_{i}-\nu_{m}\right)\left(\nu_{j}-\nu_{q}\right)\left[\left(\frac{2}{X_{1}^{2}}-\frac{2 \nu_{\ell} \nu_{p}}{X_{2}^{2}}+\frac{\nu_{p}-\nu_{\ell}}{X_{1} X_{2}}\right) w_{j} b_{q}\left(w_{i}^{\prime} b_{m}-w_{i} b_{m}^{\prime}\right)\right. \\
& \left.-\left(\frac{2}{X_{1}^{2}}-\frac{2 \nu_{\ell} \nu_{p}}{X_{2}^{2}}-\frac{\nu_{p}-\nu_{\ell}}{X_{1} X_{2}}\right) w_{i} b_{m}\left(w_{j}^{\prime} b_{q}-w_{j} b_{q}^{\prime}\right)+\frac{\nu_{\ell}-\nu_{p}}{X_{1} X_{2}}\left(\frac{\Delta_{1}}{X_{1}}-\frac{\Delta_{2}}{X_{2}}\right) w_{i} b_{m} w_{j} b_{q}\right],
\end{aligned}
$$

where the indices $p$ and $\ell$ are such that $\epsilon_{i m \ell}$ and $\epsilon_{j q p}$ do not vanish. With such an expression for $\Lambda_{m q}^{i j}$, we find that eq. (B.12) no longer holds, by numerically testing it in various 
examples of values of $b_{i}$. This proves that there is no boundary action from which the boundary condition $W+\bar{W}=0$ can be derived.

Remember however that this proof of the non-existence of the boundary action relies on our assumption that only Neumann-type boundary conditions are allowed, and Dirichlettype boundary conditions do not occur. So there is no contradiction with the special case (3.22) when Dirichlet-type conditions could be derived by varying an action (whose boundary term was actually zero). But we saw in section 4 that in the generic case the boundary condition $W+\bar{W}=0$ corresponds to two-dimensional D-branes, and we do not expect Dirichlet conditions to apply.

Open Access. This article is distributed under the terms of the Creative Commons Attribution Noncommercial License which permits any noncommercial use, distribution, and reproduction in any medium, provided the original author(s) and source are credited.

\section{References}

[1] V.A. Fateev and A.V. Litvinov, Correlation functions in conformal Toda field theory I, JHEP 11 (2007) 002 [arXiv:0709.3806] [SPIRES].

[2] A.B. Zamolodchikov, Infinite additional symmetries in two-dimensional conformal quantum field theory, Theor. Math. Phys. 65 (1985) 1205 [SPIRES].

[3] V.A. Fateev and S.L. Lukyanov, The models of two-dimensional conformal quantum field theory with $Z_{n}$ symmetry, Int. J. Mod. Phys. A 3 (1988) 507 [SPIRES].

[4] A.A. Belavin, A.M. Polyakov and A.B. Zamolodchikov, Infinite conformal symmetry in two-dimensional quantum field theory, Nucl. Phys. B 241 (1984) 333 [SPIRES].

[5] V.A. Fateev and A.B. Zamolodchikov, Conformal quantum field theory models in two-dimensions having $Z_{3}$ symmetry, Nucl. Phys. B 280 (1987) 644 [SPIRES].

[6] V.A. Fateev and A.V. Litvinov, Correlation functions in conformal Toda field theory II, JHEP 01 (2009) 033 [arXiv:0810.3020] [SPIRES].

[7] J.L. Cardy, Boundary conditions, fusion rules and the Verlinde formula, Nucl. Phys. B 324 (1989) 581 [SPIRES].

[8] P. Bouwknegt and K. Schoutens, W symmetry in conformal field theory, Phys. Rept. 223 (1993) 183 [hep-th/9210010] [SPIRES].

[9] S.L. Lukyanov, V.A. Fateev, Physics reviews: Additional symmetries and exactly soluble models in two-dimensional conformal field theory, Harwood, Chur Switzerland (1990) [Sov. Sci. Rev. A. Phys. 15 (1990) 1].

[10] P. Di Francesco, P. Mathieu, D. Sénéchal, Conformal field theory, Springer, New York U.S.A. (1997).

[11] P. Bowcock and G.M.T. Watts, Null vectors, three point and four point functions in conformal field theory, Theor. Math. Phys. 98 (1994) 350 [hep-th/9309146] [SPIRES].

[12] S. Ribault, On sl3 Knizhnik-Zamolodchikov equations and W3 null-vector equations, JHEP 10 (2009) 002 [arXiv:0811.4587] [SPIRES].

[13] A.B. Zamolodchikov and A.B. Zamolodchikov, Structure constants and conformal bootstrap in Liouville field theory, Nucl. Phys. B 477 (1996) 577 [hep-th/9506136] [SPIRES]. 
[14] A.N. Leznov and M.V. Savelev, Representation of zero curvature for the system of nonlinear partial differential equations $x_{\alpha, z \bar{z}}=\exp (k x)_{\alpha}$ and its integrability,

Lett. Math. Phys. 3 (1979) 489 [SPIRES].

[15] V. de Alfaro and A.T. Filippov, Multi-exponential models of (1+1)-dimensional dilaton gravity and Toda-Liouville integrable models, Theor. Math. Phys. 162 (2010) 34 [arXiv:0902.4445] [SPIRES].

[16] V. Fateev, A.B. Zamolodchikov and A.B. Zamolodchikov, Boundary Liouville field theory. I: Boundary state and boundary two-point function, hep-th/0001012 [SPIRES].

[17] J. Teschner, Remarks on Liouville theory with boundary, hep-th/0009138 [SPIRES].

[18] A.B. Zamolodchikov and A.B. Zamolodchikov, Liouville field theory on a pseudosphere, hep-th/0101152 [SPIRES].

[19] V.A. Fateev, Normalization factors, reflection amplitudes and integrable systems, hep-th/0103014 [SPIRES].

[20] N. Drukker, D. Gaiotto and J. Gomis, The virtue of defects in $4 D$ gauge theories and $2 D$ CFTs, arXiv: 1003.1112 [SPIRES].

[21] L. Takhtajan, A. Vinogradov, Theory of the Eisenstein series for the group $\mathrm{SL}(3, R)$ and its application to the binary problem I, Notes of the LOMI seminars 76 (1978) 5.

[22] A. Anderson, B.E.W. Nilsson, C.N. Pope and K.S. Stelle, The multivalued free field maps of Liouville and Toda gravities, Nucl. Phys. B 430 (1994) 107 [hep-th/9401007] [SPIRES]. 